\begin{tabular}{|c|c|}
\hline Title & Generalized Weak Weyl Relation and Decay of Quantum Dynamics \\
\hline Author(s) & A rai, A sao \\
\hline Citation & $\begin{array}{l}\text { Reviews in Mathematical Phy sics, 17(9), 1071-1109 } \\
\text { https://doi.org/10.1142/S0129055X 05002479 }\end{array}$ \\
\hline Issue Date & 2005 \\
\hline Doc URL & http:/hdl .handle.net/2115/1390 \\
\hline Type & article (author version) \\
\hline Note & $\begin{array}{l}\text { Reprinted from Publication, Reviews in Mathematical Physics, Vol 17-9, A rai, A, Generalized Weak Weyl Relation and } \\
\text { Decay of Quantum Dynamics, p.1071-1109, c2005, with permission from W orld Scientific Publishing Co. Pte. Ltd, } \\
\text { Singapore }\end{array}$ \\
\hline File Information & RMP17-9.pdf \\
\hline
\end{tabular}

Instructions for use 


\title{
Generalized Weak Weyl Relation and Decay of Quantum Dynamics
}

\author{
Asao Arai \\ Department of Mathematics, Hokkaido University \\ Sapporo 060-0810, Japan \\ E-mail: arai@math.sci.hokudai.ac.jp
}

January 30, 2006

This paper was published in Reviews in Mathematical Physics Vol. 17 (2005), 10711109.

\begin{abstract}
Let $H$ be a self-adjoint operator on a Hilbert space $\mathcal{H}, T$ be a symmetric operator on $\mathcal{H}$ and $K(t)(t \in \mathbb{R})$ be a bounded self-adjoint operator on $\mathcal{H}$. We say that ( $T, H, K$ ) obeys the generalized weak Weyl relation (GWWR) if $e^{-i t H} D(T) \subset D(T)$ for all $t \in \mathbb{R}$ and $T e^{-i t H} \psi=e^{-i t H}(T+K(t)) \psi, \forall \psi \in D(T)$ ( $D(T)$ denotes the domain of $T$ ). In the context of quantum mechanics where $H$ is the Hamiltonian of a quantum system, we call $T$ a generalized time opeartor of $H$. We first investigate, in an abstract framework, mathematical structures and properties of triples $(T, H, K)$ obeying the GWWR. These include the absolute continuity of the spectrum of $H$ restricted to a closed subspace of $\mathcal{H}$, an uncertainty relation between $H$ and $T$ (a "time-energy uncertainty relation"), the decay property of transition probabilities $\left|\left\langle\psi, e^{-i t H} \phi\right\rangle\right|^{2}$ as $|t| \rightarrow \infty$ for all vectors $\psi$ and $\phi$ in a subspace of $\mathcal{H}$, where $\langle\cdot, \cdot\rangle$ denotes the inner product of $\mathcal{H}$. We describe methods to construct various examples of triples $(T, H, K)$ obeying the GWWR. In particular we show that there exist generalized time operators of second quantization operators on Fock spaces (full Fock spaces, boson Fock spaces, fermion Fock spaces) which may have applications to quantum field models with interactions.
\end{abstract}

Keywords: Generalized weak Weyl relation; time operator; canonical commutation relation; Hamiltonian; quantum dynamics; survival probability; decay in time; time-energy uncertainty relation; Schrödinger operator; Dirac operator; Fock space; second quantization 


\section{Contents}

1 Introduction 2

2 Fundamental Properties of the GWWR 5

2.1 Elementary facts . . . . . . . . . . . . . . . . . . . 5

2.2 Nonself-adjointness of generalized time operators $\ldots \ldots \ldots$

2.3 Construction of triples obeying the GWWR in direct sums of Hilbert spaces 9

2.4 Perturbations . . . . . . . . . . . . . . . . 10

3 Transition Probability Amplitudes and the Point Spectra of Hamiltoni$\begin{array}{ll}\text { ans } & 11\end{array}$

4 Generalized Weak CCR and Time-Energy Uncertainty Relations 12

5 The Point Spectra of Generalized Time Operators 14

6 Commutation Formulas and Absolute Continuity 14

6.1 General cases . . . . . . . . . . . . . . . . . . . . . . . . 15

6.2 A special case . . . . . . . . . . . . . . . . . . . . . 16

7 Absence of Minimum-Uncertainty States 19

8 Power Decays of Transition Probability Amplitudes in Quantum Dynamics 21

8.1 A simple case . . . . . . . . . . . . . . . . . . . . . . . . . 22

8.2 Higher order dcays in smaller subspaces . . . . . . . . . . . . . . . . . 22

8.3 Correlation functions . . . . . . . . . . . . . . . . . . . . . 25

8.4 Heat semi-groups . . . . . . . . . . . . . . . . . . . . . 27

9 Abstract Version of Wigner's Time-Energy Uncertainty Relation 27

10 Structure producing successively triples obeying the GWWR 29

11 Generalized Time Operators of Partial Differential Operators $\quad 31$

11.1 Constructions from the Schrödinger representation of the CCR with $d$ degrees of freedom . . . . . . . . . . . . . . . . 31

11.2 Abstract Dirac operators . . . . . . . . . . . . . . . 34

12 Representations of the GWWR in Fock Spaces 35

12.1 Tensor Representations of the GWWR . . . . . . . . . . . . . . 35

12.2 Constructions of triples obeying the GWWR in Fock Spaces $\ldots . .36$

\section{Introduction}

In this paper we develop, in an abstract framework, an operator theory of a commutation relation, which is a generalization of a variant of the canonical commutation relation 
(CCR) with one degree of freedom, and put a basis for applications of the theory to quantum mechanics and quantum field theory.

To explain new features of the present work, we first recall some of the basic aspects on the representation theory of the CCR. As is well-known, a representation of the CCR with one degree of freedom is defined to be a triple $(\mathcal{H}, \mathcal{D},(Q, P))$ consisting of a complex Hilbert space $\mathcal{H}$, a dense subspace $\mathcal{D}$ of $\mathcal{H}$ and a pair $(Q, P)$ of symmetric operators on $\mathcal{H}$ such that $\mathcal{D} \subset D(Q P) \cap D(P Q)(D(\cdot)$ denotes operator domain) and

$$
Q P-P Q=i I
$$

on $\mathcal{D}$, where $i:=\sqrt{-1}$ and $I$ denotes the identity on $\mathcal{H}^{1}$. If both $Q$ and $P$ are self-adjoint and $\mathcal{D}$ is a common core of $Q$ and $P$, then we say that the representation $(\mathcal{H}, \mathcal{D},(Q, P))$ is self-adjoint ${ }^{2}$.

A typical example of self-adjoint representations of the CCR is the Schrödinger representation $\left(L^{2}(\mathbb{R}), C_{0}^{\infty}(\mathbb{R}),(q, p)\right)$ with $q$ being the multiplication operator by the function $x \in \mathbb{R}$ acting in $L^{2}(\mathbb{R})$ and $p:=-i D_{x}$ the generalized differential operator in the variable $x$ acting in $L^{2}(\mathbb{R})$.

There is a stronger form of representation of the CCR: A double $(\mathcal{H},(Q, P))$ consisting of a complex Hilbert space $\mathcal{H}$ and a pair $(Q, P)$ of self-adjoint operators on $\mathcal{H}$ is called a Weyl representation of the CCR with one degree of freedom if

$$
e^{i t Q} e^{i s P}=e^{-i t s} e^{i s P} e^{i t Q}, \quad \forall t, s \in \mathbb{R} .
$$

This relation is called the Weyl relation (e.g., [19, pp.274-275]). It is easy to see that the Schrödinger representation $\left(L^{2}(\mathbb{R}),(q, p)\right)$ is a Weyl representation. Von Neumann [16] proved that every Weyl representation on a separable Hilbert space is unitarily equivalent to a direct sum of the Schrödinger representation. This theorem - the von Neumann uniqueness theorem - implies that each Weyl representation of the CCR is a self-adjoint representation of the CCR ( for details, see, e.g., [6, 18]). But a self-adjoint representation of the CCR is not necessarily a Weyl representation of the CCR, namely there are selfadjoint representations of the CCR that are not Weyl representations (e.g., [8]). Physically interesting examples of self-adjoint representations of the CCR's with two degrees of freedom which are not necessarily unitarily equivalent to the Schrödinger representation

\footnotetext{
${ }^{1}$ One can generalize the concept of the representation of the CCR by taking the commutation relation (1.1) in the sense of sesquilinear form, i.e., $\mathcal{D} \subset D(Q) \cap D(P)$ and $\langle Q \psi, P \phi\rangle-\langle P \psi, Q \phi\rangle=i\langle\psi, \phi\rangle, \psi, \phi \in$ $\mathcal{D}$, where $\langle\cdot, \cdot\rangle$ denotes the inner product of $\mathcal{H}$.

${ }^{2}$ There are representations of the CCR which cannot be self-adjoint. For example, consider the Hilbert space $L^{2}\left(\mathbb{R}_{+}\right)$with $\mathbb{R}_{+}:=(0, \infty)$ and define operators $q_{+}, p_{+}$on $L^{2}\left(\mathbb{R}_{+}\right)$as follows:

$$
\begin{aligned}
& D\left(q_{+}\right):=\left\{\left.f \in L^{2}\left(\mathbb{R}_{+}\right)\left|\int_{\mathbb{R}_{+}}\right| r f(r)\right|^{2} d r<\infty\right\}, \quad\left(q_{+} f\right)(r):=r f(r), f \in D\left(q_{+}\right), \text {a.e.r } \in \mathbb{R}_{+}, \\
& D\left(p_{+}\right):=C_{0}^{\infty}\left(\mathbb{R}_{+}\right), \quad\left(p_{+} f\right)(r):=-i \frac{d f(r)}{d r}, f \in D\left(p_{+}\right), \text {a.e. } r \in \mathbb{R}_{+} .
\end{aligned}
$$

Then $q_{+}$is self-adjoint, $p$ is symmetric and $\left(L^{2}\left(\mathbb{R}_{+}\right), C_{0}^{\infty}\left(\mathbb{R}_{+}\right),\left(q_{+}, p_{+}\right)\right)$is a representation of the CCR with one degree of freedom. It is not so difficult to prove that $p_{+}$has no self-adjoint extensions (e.g., see [6, Chapter 2, Example D.1]). Therefore $\left(q_{+}, p_{+}\right)$cannot be extended to a self-adjoint representation of the CCR on $L^{2}\left(\mathbb{R}_{+}\right)$.
} 
of the CCR's appear in two-dimensional gauge quantum mechanics with singular gauge potentials. These representations, which are closely related to the so-called AharonovBohm effect [1], have been studied extensively by the present author in a series of papers (see [5] and references therein).

Schmüdgen [21] presented and studied a weaker version of the Weyl relation with one degree of freedom: Let $T$ be a symmetric operator and $H$ be a self-adjoint operator on a Hilbert space $\mathcal{H}$. We say that $(T, H)$ obeys the weak Weyl relation (WWR) if $e^{-i t H} D(T) \subset D(T)$ for all $t \in \mathbb{R}$ and

$$
T e^{-i t H} \psi=e^{-i t H}(T+t) \psi, \quad \forall \psi \in D(T), \forall t \in \mathbb{R},
$$

where, for later convenience, we use the symbols $(T, H)$ instead of $(Q, P)$. We call $(\mathcal{H},(T, H))$ a weak Weyl representation of the CCR with one degree of freedom. It is easy to see that every Weyl representation of the CCR is a weak Weyl representation of the CCR. But the converse is not true [21] ${ }^{3}$. It should be remarked also that the WWR implies the CCR, but a representation of the CCR is not necessarily a weak Weyl representation of the CCR. In this sense the WWR is between the CCR and the Weyl relation.

The WWR was used to study a time operator with application to survival probabilities in quantum dynamics $[11,12]$ (in the article [11], the WWR is called the $T$-weak Weyl relation), where $H$ is taken to be the Hamiltonian of a quantum system. It was proven in [11] that, if $(T, H)$ obeys the WWR, then $H$ has no point spectrum and its spectrum is purely absolutely continuous [11, Corollary 4.3, Theorem 4.4]. This kind class of $H$, however, is somewhat restrictive. From this point of view, it would be natural to investigate a general version of the WWR (if any) such that $H$ is not necessarily purely absolutely continuous. This is one of the motivations of the present work.

The general version of the WWR we consider in the present paper is defined as follows:

Definition 1. 1 Let $T$ be a symmetric operator on a Hilbert space $\mathcal{H}, H$ be a selfadjoint operator on $\mathcal{H}$ and $K(t)(t \in \mathbb{R})$ be a bounded self-adjoint operator on $\mathcal{H}$ with $D(K(t))=\mathcal{H}, \forall t \in \mathbb{R}$. We say that $(T, H, K)$ obeys the generalized weak Weyl relation (GWWR) in $\mathcal{H}$ if $e^{-i t H} D(T) \subset D(T)$ for all $t \in \mathbb{R}$ and

$$
T e^{-i t H} \psi=e^{-i t H}(T+K(t)) \psi, \quad \forall \psi \in D(T), \forall t \in \mathbb{R} .
$$

We call the operator-valued function $K$ the commutation factor in the GWWR. Also we sometimes say that $(T, H, K)$ is a representation of the GWWR.

Obviously the case $K(t)=t$ in the GWWR gives the WWR. Hence the GWWR is certainly a generalization of the WWR. One can show also that the GWWR implies a generalized version of the CCR (Proposition 4.3 in the present paper). Since the $(\mathrm{G}) \mathrm{WWR}$ is a weaker version of the Weyl relation, the strong properties arising from the Weyl relation (e.g., spectral properties) may be weakend by the $(G) W W R$. It is very interesting to investigate this aspect. Thus triples $(T, H, K)$ obeying the GWWR become

\footnotetext{
${ }^{3}$ The pair $\left(p_{+},-q_{+}\right)$in the preceding footnote obeys the WWR. But it cannot be a Weyl representation, since $p_{+}$is not self-adjoint (or $-q_{+}$is nonpositive).
} 
the main objects of the present paper. As suggested above, in applications to quantum mechanics and quantum field theory, we have in mind the case where $H$ is the Hamiltonian of a quantum system. In this realization of $H$, we call $T$ a generalized time operator. We show that the GWWR implies a "time-energy uncertainty relation" between $H$ and $T$ ( for physical discussions related to this aspect, see [15] and references therein). Mathematically rigorous studies for time-energy uncertainty relations, which, however, do not use time operators, are given in [17]. In the present paper we construct generalized time operators for Hamiltonians in both relativistic and nonrelativistic quantum mechanics including Dirac type operators as well as in quantum field theory.

The outline of the present paper is as follows. In Section 2 we discuss fundamental properties of representations $(T, H, K)$ of the GWWR. In Section 3 we derive a decay property (in time $t \in \mathbb{R}$ ) of transition probability amplitudes $\left\langle\psi, e^{-i t H} \phi\right\rangle$ for vectors $\psi, \phi$ in a suitable subspace, where $\langle\cdot, \cdot\rangle$ denotes the inner product of $\mathcal{H}$. In Section 4 , we introduce a concept of the generalized weak CCR and show that the GWWR implies the generalized weak CCR. We derive also a time-energy uncertainty relation. Section 5 is concerned with properties of the point spectrum of $T$. In Section 6 we develop functional calculus for the GWWR. In a special case where $K(t)=K_{C}(t):=t C$ with $C$ a bounded self-adjoint operator, we prove the absolute continuity of $H$ restricted to the closure of $\operatorname{Ran}(C)$, the range of $C$. In Section 7, we prove absence of minimum-uncertainty states for each representation $\left(T, H, K_{C}\right)$ with $T$ being closed and $H$ being bounded from below. This is an interesting aspect in the sense that it shows a difference from the Weyl representation (on a separable Hilbert space) or the Fock representation of the CCR in which minimum-uncertainty states exist (see Remark 7.1 for more details). In Section 8, for representations $\left(T, H, K_{C}\right)$ of the GWWR, we derive power laws for decays (in time) of transition probability amplitudes as well as two-point correlation functions. Heat semigroups $e^{-\beta H}(\beta>0)$ generated by $H$ (under the condition that $H$ is bounded from below) are also considered. In Section 9 we present an abstract version of Wigner's time-energy uncertainty relation [23]. In Section10, we show that there exists an structure producing successively representations of the GWWR. In Section 11, we construct concrete classes of representations of the GWWR, using partial differential operators acting in $L^{2}\left(\mathbb{R}^{d}\right)$ $(d \in \mathbb{N})$. We also find generalized time operators for an abstract Dirac operator. In the last section we present a tensor representation of the GWWR and construct generalized time operators for second quantization operators on Fock spaces (full Fock spaces, boson Fock spaces, fermion Fock spaces). This puts a basis to investigations of quantum field models with interactions.

\section{Fundamental Properties of the GWWR}

Throughout this section, we assume that $(T, H, K)$ obeys the GWWR in a Hilbert space $\mathcal{H}$ (Definition 1.1). We denote the inner product and the norm of $\mathcal{H}$ by $\langle\cdot, \cdot\rangle$ and $\|\cdot\|$ respectively.

\subsection{Elementary facts}


Proposition 2. 1 For all $t \in \mathbb{R}, e^{-i t H} D(T)=D(T)$ and the operator equality

$$
T e^{-i t H}=e^{-i t H}(T+K(t))
$$

holds. Moreover

$$
K(0)=0
$$

Proof: Taking $-t$ as the $t$ in Definition 1. 1, we have $e^{i t H} D(T) \subset D(T)$ for all $t \in \mathbb{R}$. Hence $D(T) \subset e^{-i t H} D(T) \subset D(T)$ for all $t \in \mathbb{R}$, which implies that $e^{-i t H} D(T)=D(T)$ for all $t \in \mathbb{R}$. By definition, we have $e^{-i t H}(T+K(t)) \subset T e^{-i t H}$ for all $t \in \mathbb{R}$. On the other hand, the preceding result implies that $D\left(T e^{-i t H}\right)=D(T)$ for all $t \in \mathbb{R}$. Thus $(2.1)$ follows. Letting $t=0$ in $(2.1)$, we have $K(0)=0$ on $D(T)$. Since $D(T)$ is dense and $K(0)$ is bounded, we obtain (2.2).

Proposition 2.2 Let $\bar{T}$ be the closure of $T$. Then $(\bar{T}, H, K)$ obeys the $G W W R$.

Proof: For each $\psi \in D(\bar{T})$, there exists a sequence $\left\{\psi_{n}\right\}_{n=1}^{\infty} \subset D(T)$ such that $\lim _{n \rightarrow \infty} \psi_{n}=\psi$ and $\lim _{n \rightarrow \infty} T \psi_{n}=\bar{T} \psi$. We have $T e^{-i t H} \psi_{n}=e^{-i t H}(T+K(t)) \psi_{n}$. The right hand side (r.h.s.) strongly converges to $e^{-i t H}(\bar{T}+K(t)) \psi$ as $n \rightarrow \infty$. We have $e^{-i t H} \psi_{n} \rightarrow e^{-i t H} \psi$ as $n \rightarrow \infty$. Hence $e^{-i t H} \psi \in D(\bar{T})$ and $\bar{T} e^{-i t H} \psi=e^{-i t H}(\bar{T}+K(t)) \psi$. Thus $(\bar{T}, H, K)$ obeys the GWWR.

For a linear operator $A$, we denote by $\sigma(A)$ (resp. $\sigma_{\mathrm{p}}(A)$ ) the spectrum (resp. the point spectrum) of $A$.

Corollary 2.3 For all $t \in \mathbb{R}, \sigma(T+K(t))=\sigma(T)$ and $\sigma_{\mathrm{p}}(T+K(t))=\sigma_{\mathrm{p}}(T)$, where the multiplicity of each $\lambda \in \sigma_{\mathrm{p}}(T)$ is equal to that of $\lambda \in \sigma_{\mathrm{p}}(T+K(t))$.

Proof: Operator equality (2.1) means the unitary equivalence of $T+K(t)$ and $T$. Hence, by a general theorem, the desired results follow.

Definition 2.4 We say that a linear operator $L$ on $\mathcal{H}$ strongly commutes with $H$ if $e^{-i t H} D(L) \subset D(L)$ for all $t \in \mathbb{R}$ and $e^{-i t H} L \subset L e^{-i t H}$.

Remark 2. 1 In the same way as in the proof of Proposition 2.1, one can show that $L$ strongly commutes with $H$ if and only if operator equality $e^{-i t H} L=L e^{-i t H}$ holds for all $t \in \mathbb{R}$.

Proposition 2.5 Let $S$ be a symmetric operator on $\mathcal{H}$ strongly commuting with $H$ such that $D(S) \cap D(T)$ is dense (hence $T+S$ is a symmetric operator with $D(T+S):=$ $D(T) \cap D(S))$. Then $(T+S, H, K)$ obeys the $G W W R$.

Proof: A simple calculation.

We denote by $\mathrm{B}(\mathcal{H})$ the Banach space of all bounded linear operators on $\mathcal{H}$ with domains equal to $\mathcal{H}$. 
Proposition 2.6 For all $t \in \mathbb{R}$,

$$
e^{i t H} K(-t)+K(t) e^{i t H}=0 .
$$

In particular

$$
\sigma(K(t))=\sigma(-K(-t)), \quad \sigma_{\mathrm{p}}(K(t))=\sigma_{\mathrm{p}}(-K(-t)), \quad \forall t \in \mathbb{R} .
$$

Proof: Let $t \in \mathbb{R}$. In general, for all $W \in \mathrm{B}(\mathcal{H})$ and every densely defined linear operator $A$ on $\mathcal{H}$, we have $(W A)^{*}=A^{*} W^{*}$ (operator equality). Hence, by taking the adjoint of (2.1), we have $e^{i t H} T^{*} \subset\left(T^{*}+K(t)\right) e^{i t H}$ for all $t \in \mathbb{R}$. Hence, for all $\psi \in D(T)$,

$$
e^{i t H} T \psi=(T+K(t)) e^{i t H} \psi=e^{i t H}(T+K(-t)) \psi+K(t) e^{i t H} \psi,
$$

where we have used (2.1) to obtain the second equality. This implies that $e^{i t H} K(-t) \psi+$ $K(t) e^{i t H} \psi=0$ for all $t \in \mathbb{R}$. Since $D(T)$ is dense, we obtain (2.3). Operator equality (2.3) implies the unitary equivalence of $K(t)$ and $-K(-t)$. Hence (2.4) follows.

\subsection{Nonself-adjointness of generalized time operators}

In this subsection we prove the following theorem:

Theorem 2.7 Assume that $K: \mathbb{R} \rightarrow \mathrm{B}(\mathcal{H})$ is strongly differentiable on $\mathbb{R}$ and let

$$
K^{\prime}(t):=\mathrm{s}-\frac{d K(t)}{d t},
$$

the strong derivative of $K$ in $t \in \mathbb{R}$. Suppose that $K^{\prime}(0) \neq 0, H$ is semi-bounded (i.e., bounded from below or bounded from above) and

$$
K(t) T \subset T K(t)
$$

for all $t \in \mathbb{R}$. Then $T$ is not self-adjoint.

Remark 2. 2 In the simple case $K(t)=t$, the fact stated in Theorem 2. 7 has been pointed out in [11].

We need some preliminary results.

Proposition 2.8 Suppose that $T$ is self-adjoint. Then, for all $t \in \mathbb{R}, T+K(t)$ is self-adjoint and

$$
e^{-i s T} e^{-i t H}=e^{-i t H} e^{-i s(T+K(t))}, \quad \forall s, \forall t \in \mathbb{R} .
$$

Proof: The self-adjointness of $T+K(t)$ follows from a simple application of the Kato-Rellich theorem, since $K(t)$ is bounded and self-adjoint. By (2.1), we have

$$
e^{i t H} T e^{-i t H}=T+K(t)
$$

as operator equality. Hence, by the functional calculus, we have for all $s, t \in \mathbb{R}$

$$
e^{i t H} e^{-i s T} e^{-i t H}=e^{-i s(T+K(t))},
$$

which is equivalent to $(2.7)$. 
Definition 2.9 We say that two self-adjoint operators $A$ and $B$ on a Hilbert space strongly commute if their spectral measures commute : $E_{A}\left(J_{1}\right) E_{B}\left(J_{2}\right)=E_{B}\left(J_{2}\right) E_{A}\left(J_{1}\right)$ for all Borel sets $J_{1}, J_{2}$ in $\mathbb{R}$, where $E_{A}$ (resp. $E_{B}$ ) denotes the spectral measure of $A$ (resp. B).

For characterizations of the strong commutativity of two self-adjoint operators, we refer the reader to [19, Theorem VIII.13].

Lemma 2.10 Let $A$ and $B$ be self-adjoint operators on a Hilbert space. Suppose that $B$ is bounded and $B A \subset A B$. Then $A$ and $B$ strongly commute.

Proof: The assumption implies that $B D(A) \subset D(A)$ and $B A \psi=A B \psi, \forall \psi \in D(A)$. This implies that, for all $n \in \mathbb{N}$ and $\psi \in D(A), B^{n} \psi \in D(A)$ and $B^{n} A \psi=A B^{n} \psi$. For $N \in \mathbb{N}$ and $t \in \mathbb{R}$, we set $e_{N}:=\sum_{n=0}^{N}(i t)^{n} B^{n} / n$ !. let $\psi \in D(A)$. Then $e_{N} A \psi=A e_{N} \psi$. It is easy to see that, for all $\phi \in \mathcal{H}, e_{N} \phi \rightarrow e^{i t B} \phi$ as $N \rightarrow \infty$. Hence $A e_{N} \psi \rightarrow e^{i t B} A \psi$ as $N \rightarrow \infty$. Since $A$ is closed, it follows that $e^{i t B} \psi \in D(A)$ and $A e^{i t B} \psi=e^{i t B} A \psi$. In particular, $e^{i t B} D(A) \subset D(A)$ for all $t \in \mathbb{R}$ and hence, in fact, $e^{i t B} D(A)=D(A)$. Therefore opeartor equality $e^{i t B} A e^{-i t B}=A$ follows. By the functional calculus, we have $e^{i t B} e^{i s A} e^{-i t B}=e^{i s A}, s, t \in \mathbb{R}$. This implies the strong commutativity of $A$ and $B$ (apply [19, Theorem VIII.13]).

Lemma 2.11 Let $A$ and $B$ be strongly commuting self-adjoint operators on a Hilbert space. Then $A+B$ is essentially self-adjoint and, for all $t \in \mathbb{R}$,

$$
e^{i t \overline{(A+B)}}=e^{i t A} e^{i t B}=e^{i t B} e^{i t A} .
$$

Proof: The essential self-adjointness of $A+B$ follows from the two variable functional calculus (note that $\overline{A+B}=\int_{\mathbb{R}^{2}}(\lambda+\mu) d E_{A, B}(\lambda, \mu)$, where $E_{A, B}$ is the two dimensional spectral measure such that $E_{A, B}\left(J_{1} \times J_{2}\right)=E_{A}\left(J_{1}\right) E_{B}\left(J_{2}\right)$ for all Borel sets $J_{1}, J_{2}$ in $\left.\mathbb{R}\right)$. Formula (2.9) follows from the Trotter product formula [19, Theorem VIII.31] and [19, Theorem VIII.13(c)].

\section{Proof of Theorem 2. 7}

Suppose that $T$ were self-adjoint. Then it follows from (2.6) and Lemma 2.10 that $K(t)$ and $T$ strongly commute. Hence, by (2.7) and Lemma 2.11, we have for all $s \in$ $\mathbb{R}, t \in \mathbb{R} \backslash\{0\}$

$$
\left(\frac{e^{-i t H}-1}{t}\right) e^{-i s T} \psi=e^{-i s T}\left(\frac{e^{-i t H}-1}{t}\right) \psi-e^{-i t H} e^{-i s T}\left(\frac{e^{-i s K(t)}-1}{t}\right) \psi
$$

for all $\psi \in \mathcal{H}$. We can write

$$
\frac{e^{-i s K(t)}-1}{t}=-i s \frac{K(t)}{t}+M_{s}(t)
$$

with

$$
M_{s}(t):=\sum_{n=2}^{\infty} \frac{(-i s)^{n}}{n !} \frac{K(t)}{t} K(t)^{n-1}
$$


in the operator norm topology. By the strong differentiability of $K$ with $K(0)=0$ (Proposition 2.1) and the principle of uniform boundedness, we have for each $\delta>0$

$$
C_{\delta}:=\sup _{|t|<\delta}\|K(t) / t\|<\infty, \quad D_{\delta}:=\sup _{|t|<\delta}\|K(t)\|<\infty,
$$

where, for a bounded operator $A \in \mathrm{B}(\mathcal{H}),\|A\|$ denotes the operator norm of $A$. Hence, for all $\psi \in \mathcal{H}$ and $0<|t|<\delta$,

$$
\left\|M_{s}(t) \psi\right\| \leq C_{\delta}\left(\sum_{n=2}^{\infty} \frac{|s|^{n}}{n !} D_{\delta}^{n-2}\right)\|K(t) \psi\| .
$$

Using this estimate and the fact that $K(0)=0$, we obtain $\lim _{t \rightarrow 0} M_{s}(t) \psi=0$. Hecne

$$
\lim _{t \rightarrow 0} \frac{e^{-i s K(t)}-1}{t} \psi=-i s K^{\prime}(0) \psi
$$

Let $\psi \in D(H)$. Then, by (2.10),

$$
\lim _{t \rightarrow 0}\left(\frac{e^{-i t H}-1}{t}\right) e^{-i s T} \psi=e^{-i s T}(-i H) \psi-e^{-i s T}(-i s) K^{\prime}(0) \psi,
$$

which implies that $e^{-i s T} \psi \in D(H)$ and

$$
(-i H) e^{-i s T} \psi=e^{-i s T}(-i H) \psi-e^{-i s T}(-i s) K^{\prime}(0) \psi,
$$

i.e.,

$$
e^{i s T} H e^{-i s T} \psi=H \psi-s K^{\prime}(0) \psi
$$

Hence

$$
\left\langle e^{-i s T} \psi, H e^{-i s T} \psi\right\rangle=\langle\psi, H \psi\rangle-s\left\langle\psi, K^{\prime}(0) \psi\right\rangle .
$$

Since $K^{\prime}(0)$ is a non-zero bounded self-adjoint operator by the present assumption, there exists a vector $\eta \in D(H)$ such that $\|\eta\|=1$ and $\left\langle\eta, K^{\prime}(0) \eta\right\rangle \neq 0$. Equation (2.11) implies that

$$
\sup _{s \in \mathbb{R}}\left\langle e^{-i s T} \eta, H e^{-i s T} \eta\right\rangle=\infty, \quad \inf _{s \in \mathbb{R}}\left\langle e^{-i s T} \eta, H e^{-i s T} \eta\right\rangle=-\infty .
$$

Hence $H$ is not semi-bounded (note that $\left\|e^{-i s T} \eta\right\|=1$ for all $s \in \mathbb{R}$ ). Thus we are led to a contradiction.

\subsection{Construction of triples obeying the GWWR in direct sums of Hilbert spaces}

Let $\mathcal{H}_{1}$ be a Hilbert space and $\mathcal{F}:=\mathcal{H} \oplus \mathcal{H}_{1}$. Let $\left(T_{1}, H_{1}, K_{1}\right)$ be a triple obeying the GWWR in $\mathcal{H}_{1}$. We define

$$
\widetilde{H}:=H \oplus H_{1}=\left(\begin{array}{cc}
H & 0 \\
0 & H_{1}
\end{array}\right)
$$


Proposition 2.12 Let $A$ be a bounded linear operator from $\mathcal{H}$ to $\mathcal{H}_{1}$ with $D(A)=\mathcal{H}$ and

$$
\widetilde{T}:=\left(\begin{array}{cc}
T & A^{*} \\
A & T_{1}
\end{array}\right), \quad \widetilde{K}(t):=\left(\begin{array}{cc}
K(t) & e^{i t H} A^{*} e^{-i t H_{1}}-A^{*} \\
e^{i t H_{1}} A e^{-i t H}-A & K_{1}(t)
\end{array}\right) .
$$

Then $(\widetilde{T}, \widetilde{H}, \widetilde{K})$ obeys the GWWR in $\mathcal{F}$.

Proof: By the functional calculus, we have $e^{-i t \widetilde{H}}=e^{-i t H} \oplus e^{-i t H_{1}}$ for all $t \in \mathbb{R}$. Then direct computations yield the desired result.

Note that, in Proposition 2.12, $\widetilde{T}$ is not diagonal if $A \neq 0$. This procedure of construction of a new triple obeying the GWWR obviously yields an algorithm to obtain a triple obeying the GWWR in the $N$ direct sum $\oplus_{n=1}^{N} \mathcal{H}_{n}$ of Hilbert spaces $\mathcal{H}_{n}(N \geq 2)$, provided that, for each $n$, a triple $\left(T_{n}, H_{n}, K_{n}\right)$ obeying the GWWR in $\mathcal{H}_{n}$ is given.

\subsection{Perturbations}

Let $V$ be a symmetric operator on $\mathcal{H}$ and assume that

$$
H(V):=H+V
$$

is essentially self-adjoint. It is an interesting problem to investigate if there exist a symmetric operator $T_{V}$ and an operator-valued function $K_{V}: \mathbb{R} \rightarrow \mathrm{B}(\mathcal{H})$ such that $\left(T_{V}, \overline{H(V)}, K_{V}\right)$ obeys the GWWR. Here we present only an abstract answer to this problem.

Proposition 2.13 Assume that the following conditions (i)-(iii) hold:

(i) The operators $T, H$ and $K(t)(t \in \mathbb{R})$ are reduced by a closed subspace $\mathcal{M}$ of $\mathcal{H}$. We denote their reduced part by $T_{\mathcal{M}}, H_{\mathcal{M}}$ and $K_{\mathcal{M}}(t)$ respectively.

(ii) The operator $\overline{H(V)}$ is reduced by a closed subspace $\mathcal{N}$ of $\mathcal{H}$.

(iii) There exists a unitary operator $U: \mathcal{M} \rightarrow \mathcal{N}$ such that $U H_{\mathcal{M}} U^{-1}=\overline{H(V)}_{\mathcal{N}}$.

Let

$$
T_{V}:=\left(U T_{\mathcal{M}} U^{-1}\right) \oplus 0, \quad K_{V}(t):=\left(U K_{\mathcal{M}}(t) U^{-1}\right) \oplus 0
$$

relative to the orthogonal decomposition $\mathcal{H}=\mathcal{N} \oplus \mathcal{N}^{\perp}$. Then $\left(T_{V}, \overline{H(V)}, K_{V}\right)$ obeys the GWWR.

Proof: It is obvious that $T_{V}$ is symmetric and $K_{V}(t)$ is a bounded self-adjoint operator. By direct computations, one sees that $\left(T_{V}, \overline{H(V)}, K_{V}\right)$ obeys the GWWR.

Remark 2.3 A method to find the unitary operator $U$ in Proposition 2.13 is to use the method of wave operators with respect to the pair $(H, \overline{H(V)})$. In that case, $U$ would be one of the wave operators $W_{ \pm}:=\mathrm{s}-\lim _{t \rightarrow \pm \infty} e^{i t \overline{H(V)}} J e^{-i t H} P_{\mathrm{ac}}(H)$ (if they exist) $\left(P_{\mathrm{ac}}(H)\right.$ is the orthogonal projection onto the absolutely continuous space of $H$ and $J$ is a linear operator), $\mathcal{M}=\left(\operatorname{ker} W_{ \pm}\right)^{\perp}$ and $\mathcal{N}=\overline{\operatorname{Ran}\left(W_{ \pm}\right)}$(e.g., [9, §4.2], [20, p.34, Proposition 4]). This method was taken in $[11,12]$ in the case where $H$ is the 1 -dimensional Laplacian and $V$ is a real-valued function on $\mathbb{R}$ (hence $H(V)$ is a one-dimensional Schrödinger operator). In the present paper we do not develop this aspect. 


\section{Transition Probability Amplitudes and the Point Spectra of Hamiltonians}

If a self-adjoint operator $H$ on a Hilbert space represents the Hamiltonian of a quantum system, then the transition probability amplitude of an initial state $\psi \in \mathcal{H}$ with $\|\psi\|=1$ to a state $\phi \in \mathcal{H}$ with $\|\phi\|=1$ at time $t \in \mathbb{R}$ is given by $\left\langle\phi, e^{-i t H} \psi\right\rangle$. The square $\left|\left\langle\phi, e^{-i t H} \psi\right\rangle\right|^{2}$ of its modulus is called the transition probability from $\psi$ at time 0 to $\phi$ at time $t$. In particular $\left|\left\langle\psi, e^{-i t H} \psi\right\rangle\right|^{2}$ is referred to as the survival probability, at time $t$, of the state $\psi$.

Let $(T, H, K)$ be a triple obeying the GWWR in a Hilbert space $\mathcal{H}$. The following proposition is concerned with upper bounds of the modulus of a transition probabilty amplitude in time $t$.

Proposition 3.1 Suppose that there is a constant $\alpha>0$ such that the strong limit

$$
L_{\alpha}:=\mathrm{s}-\lim _{t \rightarrow \infty} \frac{K(t)}{t^{\alpha}} \in \mathrm{B}(\mathcal{H})
$$

exists. Let $S$ be a symmetric operator strongly commuting with $H$. Then, for all $\psi, \phi \in$ $D(T) \cap D(S)$ and $t>0$,

$$
\left|\left\langle\psi, e^{-i t H} L_{\alpha} \phi\right\rangle\right| \leq \frac{\|(T+S) \psi\|\|\phi\|+\|\psi\|\|(T+S) \phi\|}{t^{\alpha}}+\|\psi\|\left\|\left(L_{\alpha}-\frac{K(t)}{t^{\alpha}}\right) \phi\right\| .
$$

Proof: Let $L=L_{\alpha}$ and $L(t):=K(t) / t^{\alpha}$. Then

$$
\begin{aligned}
\left|\left\langle\psi, e^{-i t H} L \phi\right\rangle\right| & \leq\left|\left\langle\psi, e^{-i t H}(L-L(t)) \phi\right\rangle\right|+\left|\left\langle\psi, e^{-i t H} L(t) \phi\right\rangle\right| \\
& \leq\|\psi\|\|(L-L(t)) \phi\|+\left|\left\langle\psi, e^{-i t H} L(t) \phi\right\rangle\right| .
\end{aligned}
$$

By Proposition 2.5, we have $e^{-i t H} L(t) \phi=t^{-\alpha}\left[(T+S) e^{-i t H} \phi-e^{-i t H}(T+S) \phi\right]$. Using this relation, we have

$$
\left|\left\langle\psi, e^{-i t H} L(t) \phi\right\rangle\right| \leq \frac{\|(T+S) \psi\|\|\phi\|+\|\psi\|\|(T+S) \phi\|}{t^{\alpha}} .
$$

Hence (3.2) follows.

Remark 3. 1 Proposition 3. 1 is a generalization of [11, Theorem 4.1] where the special case $K(t)=t$ is considered.

The following corollary is a generalized version of [11, Corollary 4.3]:

Corollary 3.2 Suppose that the assumption of Proposition 3.1 holds. Then, for all $\psi, \phi \in \mathcal{H}$,

$$
\lim _{t \rightarrow \infty}\left\langle\psi, e^{-i t H} L_{\alpha} \phi\right\rangle=0 .
$$


Proof: The proof is similar to that of [11, Corollary 4.3]. By the polarization identity, we need only to prove (3.3) with $\phi=\psi$. Since $D(T)$ is dense, there exists a sequence $\psi_{n} \in D(T)$ such that $\lim _{n \rightarrow \infty} \psi_{n}=\psi$. Then, in the same way as in the proof of the preceding proposition, we have

$$
\begin{gathered}
\left|\left\langle\psi, e^{-i t H} L_{\alpha} \psi\right\rangle\right| \leq\left\|\psi-\psi_{n}\right\|\left\|L_{\alpha} \psi\right\|+\left\|\psi_{n}\right\|\left\|L_{\alpha}\right\|\left\|\psi-\psi_{n}\right\| \\
+\left|\left\langle\psi_{n}, e^{-i t H} L_{\alpha} \psi_{n}\right\rangle\right|
\end{gathered}
$$

By (3.2) with $\psi=\phi=\psi_{n}$ and $S=0$, we have $\lim _{t \rightarrow \infty}\left|\left\langle\psi_{n}, e^{-i t H} L_{\alpha} \psi_{n}\right\rangle\right|=0$. Hence

$$
\limsup _{t \rightarrow \infty}\left|\left\langle\psi, e^{-i t H} L_{\alpha} \psi\right\rangle\right| \leq\left\|\psi-\psi_{n}\right\|\left\|L_{\alpha} \psi\right\|+\left\|\psi_{n}\right\|\left\|L_{\alpha}\right\|\left\|\psi-\psi_{n}\right\| .
$$

Then, taking $n \rightarrow \infty$, we obtain (3.3) with $\phi=\psi$.

This corollary implies an interesting structure of the point spectrum of $H$ :

Corollary 3. 3 Suppose that the assumption of Proposition 3. 1 holds. Then, for all $E \in \mathbb{R}, \operatorname{ker}(H-E) \subset \operatorname{ker} L_{\alpha}$. In particular, if $\operatorname{ker} L_{\alpha}=\{0\}$, then $\sigma_{\mathrm{p}}(H)=\emptyset$.

Proof: Let $\psi_{E} \in \operatorname{ker}(H-E)$. Then $e^{i t H} \psi_{E}=e^{i t E} \psi_{E}$. Taking $\psi=\psi_{E}$ in (3.3), we obtain $\left\langle\psi_{E}, L_{\alpha} \phi\right\rangle=0$ for all $\phi \in \mathcal{H}$. This implies that $L_{\alpha} \psi_{E}=0$, i.e., $\psi_{E} \in \operatorname{ker} L_{\alpha}$.

Remark 3.2 Corollary 3.3 is a generalization of [11, Corollary 4.3] where the case $K(t)=t$ is considered.

\section{Generalized Weak CCR and Time-Energy Uncer- tainty Relations}

Let $A, B$ be symmetric operators on a Hilbert space $\mathcal{H}$ and $C \in \mathrm{B}(\mathcal{H})$ be a self-adjoint operator. We say that $(A, B, C)$ obeys the generalized weak $C C R$ (GWCCR) if

$$
\langle A \psi, B \phi\rangle-\langle B \psi, A \phi\rangle=\langle\psi, i C \phi\rangle, \quad \forall \psi, \phi \in D(A) \cap D(B) .
$$

The case $C=I$ (the identity on $\mathcal{H}$ ) is the usual CCR with one degree of freedom in the sense of sesquilinear form.

For a symmetric operator $A$ on a Hilbert space, a constant $a \in \mathbb{R}$ and a unit vector $\psi \in D(A)$, we define

$$
(\Delta A)_{\psi}(a):=\|(A-a) \psi\|,
$$

an uncertainty of $A$ in the state vector $\psi$. The quantity $(\Delta A)_{\psi}(a)$ with $a=\langle\psi, A \psi\rangle$ is the usual uncertainty of $A$ in the state vector $\psi$. We set

$$
(\Delta A)_{\psi}:=(\Delta A)_{\psi}(\langle\psi, A \psi\rangle) .
$$

We also introduce

$$
\delta_{C}:=\inf _{\psi \in(\operatorname{ker} C)^{\perp},\|\psi\|=1}|\langle\psi, C \psi\rangle| .
$$


Proposition 4. 1 Suppose that $(A, B, C)$ obeys the $G W C C R$. Then, for all $\psi \in D(A) \cap$ $D(B) \cap(\operatorname{ker} C)^{\perp}$ with $\|\psi\|=1$ and all $a, b \in \mathbb{R}$,

$$
(\Delta A)_{\psi}(a)(\Delta B)_{\psi}(b) \geq \frac{\delta_{C}}{2} .
$$

Proof: It is easy to see that $(A-a, B-b, C)$ also obeys the GWCCR. Hence, applying the Cauchy-Schwarz inequality on a non-negative, sesquilinear form on a space of linear operators [17, Lemma 1], we have for all $\psi \in D(A) \cap D(B)$

$$
(\Delta A)_{\psi}(a)(\Delta B)_{\psi}(b) \geq \frac{1}{2}|\langle\psi, C \psi\rangle| .
$$

Thus (4.5) follows.

Proposition 4. 2 Suppose that $(A, B, C)$ obeys the $G W C C R$ with $C \geq 0$. Then, for all $\lambda \in \mathbb{R}, \operatorname{ker}(B-\lambda) \cap D(A) \subset \operatorname{ker} C$ and $\operatorname{ker}(A-\lambda) \cap D(B) \subset \operatorname{ker} C$.

Proof: Let $\psi \in \operatorname{ker}(B-\lambda) \cap D(A)$. Then, taking $\phi=\psi$ in (4.1), we have $\langle\psi, C \psi\rangle=0$. Since $C$ is nonnegative, it follows that $C \psi=0$, i.e., $\psi \in \operatorname{ker} C$.

The following proposition gives a connection of the GWWR with the GWCCR:

Proposition 4. 3 Let $(T, H, K)$ be a triple obeying the $G W W R$ in $\mathcal{H}$. Assume that $K$ is strongly differentiable on $\mathbb{R}$. Then $\left(T, H, K^{\prime}(0)\right)$ obeys the $G W C C R$ :

$$
\langle T \psi, H \phi\rangle-\langle H \psi, T \phi\rangle=\left\langle\psi, i K^{\prime}(0) \phi\right\rangle, \quad \psi, \phi \in D(T) \cap D(H) .
$$

Proof: Let $\psi, \phi \in D(T) \cap D(H)$. Then we have by (1.2)

$$
\left\langle T \psi, e^{-i t H} \phi\right\rangle=\left\langle e^{i t H} \psi, T \phi\right\rangle+\left\langle e^{i t H} \psi, K(t) \phi\right\rangle .
$$

It is well known that, for all $\eta \in D(H), e^{i s H} \eta$ is strongly differentiable with s- $d e^{i s H} \eta / d s=$ $i H e^{i s H} \eta=i e^{i s H} H \eta$. Hence the both sides of (4.7) are differentiable in $t$. Evaluating the derivatives at $t=0$ and using (2.2), we obtain (4.6).

Propositions 4.3 and 4.1 yield the following result:

Corollary 4. 4 Suppose that the same assumption as in Proposition 4. 3 holds. Then, for all $\psi \in D(T) \cap D(H) \cap\left(\operatorname{ker} K^{\prime}(0)\right)^{\perp}$ with $\|\psi\|=1$ and all $t, E \in \mathbb{R}$,

$$
(\Delta T)_{\psi}(t)(\Delta H)_{\psi}(E) \geq \frac{\delta_{K^{\prime}(0)}}{2} .
$$

In applications to quantum theory, (4.8) gives a time-energy uncertainty relation if $H$ is the Hamiltonian of a quantum system. 


\section{The Point Spectra of Generalized Time Operators}

For a linear operator $L$ on a Hilbert apce $\mathcal{H}$, we introduce a subset of $\mathcal{H}$ :

$$
N_{0}(L):=\{\psi \in D(L) \mid\langle\psi, L \psi\rangle=0\}
$$

It is obvious that $\operatorname{ker} L \subset N_{0}(L)$.

Remark 5. 1 If $L$ is a non-negative self-adjoint operator, then $N_{0}(L)=\operatorname{ker} L$.

Proposition 5. 1 Assume that $(T, H, K)$ obeys the $G W W R$ and $K$ is strongly differentiable on $\mathbb{R}$. Then, for all $E \in \mathbb{R}$,

$$
\operatorname{ker}(T-E) \subset N_{0}\left(K^{\prime}(0)\right)
$$

Proof: Let $\psi_{0} \in \operatorname{ker}(T-E)$. Then $T \psi_{0}=E \psi_{0}$. Taking the inner product of $\psi_{0}$ with the vector obtained from the operation of $(2.1)$ to $\psi_{0}$, we have $\left\langle\psi_{0}, e^{-i t H} K(t) \psi_{0}\right\rangle=$ $0, t \in \mathbb{R}$. Dividing the both sides by $t \neq 0$ and taking the limit $t \rightarrow 0$, we obtain $\left\langle\psi_{0}, K^{\prime}(0) \psi_{0}\right\rangle=0$, where we have used (2.2). Hence $\psi_{0} \in N_{0}\left(K^{\prime}(0)\right)$. Thus (5.2) follows.

Corollary 5. 2 Assume that $(T, H, K)$ obeys the $G W W R$ and $K$ is strongly differentiable on $\mathbb{R}$. Then:

(i) If $N_{0}\left(K^{\prime}(0)\right)=\{0\}$, then $\sigma_{\mathrm{p}}(T)=\emptyset$.

(ii) If $K^{\prime}(0) \geq 0$ or $K^{\prime}(0) \leq 0$, then $\sigma_{\mathrm{p}}\left(T \mid\left[D(T) \cap\left(\operatorname{ker} K^{\prime}(0)\right)^{\perp}\right]\right)=\emptyset$.

Remark 5. 2 Corollary (5.2) is a generalization of [11, Corollary 4.2] where the case $K(t)=t$ is considered.

Remark 5. 3 It may be interesting to note that the behavior of $K(t)$ at $t=0$ and $t=\infty$ is respectively related to $\sigma_{\mathrm{p}}(T)$ (Corollary 5.2 ) and $\sigma_{\mathrm{p}}(H)$ (Corollary 3.3).

\section{Commutation Formulas and Absolute Continuity}

In this section we prove commutation relations derived from the GWWR. Moreover, in the special case where the commutation factor $K(t)$ is of the form $t C$ with $C$ a bounded self-adjoint operator, we show that $H$ is reduced by $\overline{\operatorname{Ran}(C)}(\operatorname{Ran}(C)$ denotes the range of $C$ ) and its reduced part is absolutely continuous. 


\subsection{General cases}

For $p \geq 0$, we introduce a class of Borel measurable functions on $\mathbb{R}$ :

$$
L_{p}^{1}(\mathbb{R}):=\left\{F: \mathbb{R} \rightarrow \mathbb{C}, \text { Borel measurable }\left|\int_{\mathbb{R}}\right| F(t) \mid\left(1+|t|^{p}\right) d t<\infty\right\} .
$$

It is easy to see that $L_{p}^{1}(\mathbb{R})$ includes the space $\mathcal{S}(\mathbb{R})$ of rapidly decreasing $C^{\infty}$-functions on $\mathbb{R}$.

We say that a Borel measurable function $f$ is in the set $\mathcal{M}_{p}$ if it is the Fourier transform of an element $F_{f} \in L_{p}^{1}(\mathbb{R})$ :

$$
f(\lambda)=\frac{1}{\sqrt{2 \pi}} \int_{\mathbb{R}} F_{f}(t) e^{-i t \lambda} d t, \quad \lambda \in \mathbb{R} .
$$

Note that, for each $f \in \mathcal{M}_{p}, F_{f}$ is uniquely determined. We have

$$
\mathcal{S}(\mathbb{R}) \subset \mathcal{M}_{p}
$$

Moreover, every element $f$ of $\mathcal{M}_{p}$ is bounded, $[p]$ times continuously differentiable $([p]$ denotes the largest integer not exceeding $p$ ) and, for $j=1, \cdots,[p], d^{j} f / d \lambda^{j}$ is bounded.

Let $H$ be a self-adjoint operator on a Hilbert space $\mathcal{H}$ and $S: \mathbb{R} \rightarrow \mathrm{B}(\mathcal{H})$ be Borel measurable such that, for all $\psi \in \mathcal{H}$,

$$
\|S(t) \psi\| \leq c\left(1+|t|^{p}\right)\|\psi\|, \quad t \in \mathbb{R}
$$

with constants $c>0$ and $p \geq 0$ independent of $\psi$. Then, for all $\psi \in \mathcal{H}$ and $f \in \mathcal{M}_{p}$, the strong integral

$$
f(H, S) \psi:=\frac{1}{\sqrt{2 \pi}} \int_{\mathbb{R}} F_{f}(t) e^{-i t H} S(t) \psi d t
$$

exists and $f(H, S) \in \mathrm{B}(\mathcal{H})$.

Theorem 6. 1 Assume that $(T, H, K)$ obeys the GWWR. Suppose that $K$ is strongly continuous and, for all $\psi \in \mathcal{H}$,

$$
\|K(t) \psi\| \leq c\left(1+|t|^{p}\right)\|\psi\|
$$

where $c>0$ and $p \geq 0$ are constants independent of $\psi$. Let $f \in \mathcal{M}_{p}$. Then, for all $\psi \in D(\bar{T})$, we have $f(H) \psi \in D(\bar{T})$ and

$$
\bar{T} f(H) \psi=f(H) \bar{T} \psi+f(H, K) \psi,
$$

where $f(H):=\int_{\mathbb{R}} f(\lambda) d E_{H}(\lambda)$.

Proof: Let $f \in \mathcal{M}_{p}$ and $\psi \in D\left(T^{*}\right), \phi \in D(\bar{T})$. Then, by the functional calculus of the self-adjoint operator $H$, we have

$$
\left\langle T^{*} \psi, f(H) \phi\right\rangle=\frac{1}{\sqrt{2 \pi}} \int_{\mathbb{R}} F_{f}(t)\left\langle T^{*} \psi, e^{-i t H} \phi\right\rangle d t .
$$


Since $e^{-i t H} \phi \in D(\bar{T})=D\left(T^{* *}\right)$ by Proposition 2.2, it follows that $\left\langle T^{*} \psi, e^{-i t H} \phi\right\rangle=$ $\left\langle\psi, \bar{T} e^{-i t H} \phi\right\rangle$. Using the fact that $(\bar{T}, H, K)$ obeys the GWWR (Proposition 2. 2 ), we see that the r.h.s. is equal to $\left\langle\psi, e^{-i t H} \bar{T} \phi\right\rangle+\left\langle\psi, e^{-i t H} K(t) \phi\right\rangle$. Hence we obtain

$$
\left\langle T^{*} \psi, f(H) \phi\right\rangle=\langle\psi, f(H) \bar{T} \phi\rangle+\frac{1}{\sqrt{2 \pi}} \int_{\mathbb{R}} F_{f}(t)\left\langle\psi, e^{-i t H} K(t) \phi\right\rangle d t .
$$

Therefore $\left\langle T^{*} \psi, f(H) \phi\right\rangle=\langle\psi, f(H) \bar{T} \phi+f(H, K) \phi\rangle$. Since $\psi \in D\left(T^{*}\right)$ is arbitrary, it follows that $f(H) \phi \in D\left(T^{* *}\right)=D(\bar{T})$ and (6.6) holds.

\subsection{A special case}

In this section we consider a special case of a triple $(T, H, K)$ obeying the GWWR in a Hilbert space $\mathcal{H}$ : We assume that $K$ is of the form

$$
K_{C}(t):=t C, \quad t \in \mathbb{R}
$$

with $C$ being a bounded self-adjoint operator on $\mathcal{H}$. In this case a more detailed analysis is possible as shown below.

We set

$$
\begin{aligned}
& C_{\mathrm{b}}^{1}(\mathbb{R}):= \\
& C_{\mathrm{b},+}^{1}(\mathbb{R}):=\left\{f \in C^{1}(\mathbb{R}) \mid f \text { and } f^{\prime} \text { are bounded }\right\} \\
&\left\{f \in C^{1}(\mathbb{R}) \mid \text { for some } a \in \mathbb{R}, \sup _{\lambda \geq a}|f(\lambda)|<\infty\right. \text { and } \\
&\left.\sup _{\lambda \geq a}\left|f^{\prime}(\lambda)\right|<\infty\right\} .
\end{aligned}
$$

Theorem 6.2 Let $C$ be a bounded self-adjoint operator on $\mathcal{H}$ and suppose that $(T, H$, $K_{C}$ ) obeys the $G W W R$.

(i) Let $f \in C_{\mathrm{b}}^{1}(\mathbb{R})$. Then $f(H) D(\bar{T}) \subset D(\bar{T})$ and

$$
\bar{T} f(H) \psi-f(H) \bar{T} \psi=i f^{\prime}(H) C \psi
$$

for all $\psi \in D(\bar{T})$.

(ii) Suppose that $H$ is bounded from below. Then, for all $f \in C_{\mathrm{b},+}^{1}(\mathbb{R})$, the same conclusion as that of part (i) holds. In particular, for all $z \in \mathbb{C}$ with $\Re z>0$, $e^{-z H} D(\bar{T}) \subset D(\bar{T})$ and, for all $\psi \in D(\bar{T})$

$$
\bar{T} e^{-z H} \psi-e^{-z H} \bar{T} \psi=-i z e^{-z H} C \psi .
$$

Proof: (i) We first consider the case where $f \in \mathcal{M}_{1}$. Then it is easy to see that $f\left(H, K_{C}\right)=i f^{\prime}(H) C$. By this fact and Theorem 6.1, (6.10) holds.

We next consider the case where $f$ is an arbitrary element in the set $C_{0}^{1}(\mathbb{R}):=\{f \in$ $C^{1}(\mathbb{R}) \mid \operatorname{supp} f$ is compact\}, where $\operatorname{supp} f$ means the support of $f$. For a function $g$ on $\mathbb{R}$, we set

$$
\|g\|_{\infty}:=\sup _{\lambda \in \mathbb{R}}|g(\lambda)|
$$


Using the Friedrichs mollifier, we can find a sequence $\left\{f_{n}\right\}_{n=1}^{\infty} \subset C_{0}^{\infty}(\mathbb{R})$ (the set of infinitely differentiable functions on $\mathbb{R}$ with compact support) such that $\sup _{n \geq 1}\left\|f_{n}\right\|_{\infty}<$ $\infty, \sup _{n \geq 1}\left\|f_{n}^{\prime}\right\|_{\infty}<\infty$ and, for all $\lambda \in \mathbb{R}, f_{n}(\lambda) \rightarrow f(\lambda), f_{n}^{\prime}(\lambda) \rightarrow f^{\prime}(\lambda)$ as $n \rightarrow \infty$. Then, by using the functional calculus for $H$, we see that

$$
f_{n}(H) \rightarrow f(H), \quad f_{n}^{\prime}(H) \rightarrow f^{\prime}(H)
$$

strongly as $n \rightarrow \infty$. By the fact that $C_{0}^{\infty}(\mathbb{R}) \subset \mathcal{M}_{1}$ and the preceding result, we have

$$
\bar{T} f_{n}(H) \psi=f_{n}(H) \bar{T} \psi+i f_{n}^{\prime}(H) C \psi
$$

for all $\psi \in D(\bar{T})$. Hence $\bar{T} f_{n}(H) \psi \rightarrow f(H) \bar{T} \psi+i f^{\prime}(H) C \psi$ as $n \rightarrow \infty$. Since $\bar{T}$ is closed, it follows that $f(H) \psi \in D(\bar{T})$ and (6.10) holds.

Finally we consider the case where $f \in C_{\mathrm{b}}^{1}(\mathbb{R})$. Let $\chi \in C_{0}^{\infty}(\mathbb{R})$ such that $\chi(0)=1$ and set $f_{n}(\lambda):=\chi(\lambda / n) f(\lambda)$. Then $f_{n} \in C_{0}^{1}(\mathbb{R})$ and

$$
f_{n}^{\prime}(\lambda)=\frac{1}{n} \chi^{\prime}\left(\frac{\lambda}{n}\right) f(\lambda)+\chi\left(\frac{\lambda}{n}\right) f^{\prime}(\lambda) .
$$

It is easy to see that, for all $\lambda \in \mathbb{R}, f_{n}(\lambda) \rightarrow f(\lambda), f_{n}^{\prime}(\lambda) \rightarrow f^{\prime}(\lambda)$ as $n \rightarrow \infty$ and

$$
\left|f_{n}(\lambda)\right| \leq\|\chi\|_{\infty}\|f\|_{\infty}, \quad\left|f_{n}^{\prime}(\lambda)\right| \leq\left\|\chi^{\prime}\right\|_{\infty}\|f\|_{\infty}+\|\chi\|_{\infty}\left\|f^{\prime}\right\|_{\infty}
$$

Hence, by the functional calculus for $H$, we obtain that $f_{n}(H) \rightarrow f(H), f_{n}^{\prime}(H) \rightarrow f^{\prime}(H)$ strongly as $n \rightarrow \infty$. Then, in the same manner as in the preceding paragraph, we obtain the desired conclusion.

(ii) Let $H \geq-M$ with a constant $M \geq 0$ and $f \in C_{\mathrm{b},+}^{1}(\mathbb{R})$. Let $\chi$ be as above and define $f_{n}(\lambda):=\chi(\lambda / n) f(\lambda)$. Then $f_{n} \in C_{0}^{1}(\mathbb{R})$. Hence, by part (i),

$$
\bar{T} f_{n}(H) \psi=f_{n}(H) \bar{T} \psi+i f_{n}^{\prime}(H) C \psi
$$

for all $\psi \in D(\bar{T})$. We have for all $\psi \in \mathcal{H}$

$$
\left\|f_{n}(H) \psi-f(H) \psi\right\|^{2}=\int_{[-M, \infty)} F_{n}(\lambda) d\left\|E_{H}(\lambda) \psi\right\|^{2} .
$$

with

$$
F_{n}(\lambda):=\left|\chi\left(\frac{\lambda}{n}\right)-1\right|^{2}|f(\lambda)|^{2}
$$

We have

$$
\left|F_{n}(\lambda)\right| \leq\left(\|\chi\|_{\infty}+1\right)^{2}\left(\sup _{\lambda \geq-M}|f(\lambda)|\right)^{2}, \quad \lambda \in[-M, \infty) .
$$

Hence, by the Lebesgue dominated convergence theorem, the r.h.s. of (6.13) converges to 0 as $n \rightarrow \infty$. Hence $f_{n}(H) \rightarrow f(H)$ strongly as $n \rightarrow \infty$. Similarly we can show that $f_{n}^{\prime}(H) \rightarrow f^{\prime}(H)$. Thus, in the same way as in part (i), we obtain (6.10).

If $\Re z>0(z \in \mathbb{C})$, then the function $f_{z}: \mathbb{R} \rightarrow \mathbb{C}$ defined by $f_{z}(\lambda):=e^{-z \lambda}$ is in $C_{\mathrm{b},+}^{1}(\mathbb{R})$. Hence, by applying the preceding result, we obtain (6.11). 
Remark 6. 1 Theorem 6. 2 is a slight generalization of [21, 3.1 Proposition 1].

Proposition 6. 3 Let $C$ be a bounded self-adjoint operator on $\mathcal{H}$ and suppose that $(T, H$, $\left.K_{C}\right)$ obeys the $G W W R$. Then $H$ and $C$ strongly commute.

Proof: We can apply Proposition 2.6 to obtain that $e^{-i t H} C e^{i t H}=C, \forall t \in \mathbb{R}$. This implies the strong commutativity of $H$ and $C$.

Lemma 6. 4 Let $A$ and $B$ be strongly commuting self-adjoint operators on a Hilbert space $\mathcal{H}$. Then $\overline{\operatorname{Ran}(B)}$ reduces $A$.

Proof: Since we have the orthogonal decomposition $\mathcal{H}=\operatorname{ker} B \oplus \overline{\operatorname{Ran}(B)}$, it is sufficient to prove that ker $B$ reduces $A$. Let $P$ be the orthogonal projection onto ker $B$. Then we have $P=E_{B}(\{0\})$. Hence, by the strong commutativity of $A$ and $B, e^{i t A} P=P e^{i t A}$ for all $t \in \mathbb{R}$. This implies that $P A \subset A P$. Thus ker $B$ reduces $A$.

This lemma and Proposition 6. 3 imply the following fact:

Corollary 6.5 Under the same assumption as in Proposition 6. 3, $H$ is reduced by $\overline{\operatorname{Ran}(C)}$.

As in the case of [21, 3.2 Corollary 2], we have from Theorem 6. 2 and Corollary 6. 5 the following theorem. For a self-adjoint operator $H$, we set

$$
E_{H}(\lambda):=E_{H}((-\infty, \lambda]), \quad \lambda \in \mathbb{R} .
$$

Theorem 6. 6 Suppose that $\left(T, H, K_{C}\right)$ obeys the $G W W R$. Then $H$ is reduced by $\overline{\operatorname{Ran}(C)}$ and the reduced part $H \mid \overline{\operatorname{Ran}(C)}$ is absolutely continuous. Moreover, for all $\psi, \phi \in D(\bar{T})$, the Radon-Nikodym derivative $d\left\langle\psi, E_{H}(\lambda) C \phi\right\rangle / d \lambda$ is given by

$$
\frac{d\left\langle\psi, E_{H}(\lambda) C \phi\right\rangle}{d \lambda}=i\left(\left\langle\bar{T} \psi, E_{H}(\lambda) \phi\right\rangle-\left\langle E_{H}(\lambda) \psi, \bar{T} \phi\right\rangle\right) .
$$

Proof: The reducibility of $H$ by $\overline{\operatorname{Ran}(C)}$ has already been proved in Corollary 6.5 . Let $f \in \mathcal{S}(\mathbb{R})$ and $\psi, \phi \in D(\bar{T})$. It is easy to see that $\mathcal{S}(\mathbb{R}) \subset C_{\mathrm{b}}^{1}(\mathbb{R})$. Hence, by Theorem 6. 2 , we have

$$
\langle\bar{T} \psi, f(H) \phi\rangle-\langle\psi, f(H) \bar{T} \phi\rangle=i\left\langle\psi, f^{\prime}(H) C \phi\right\rangle .
$$

Let $\mu(\lambda):=\left\langle\psi, E_{H}(\lambda) C \phi\right\rangle$ and $\sigma(\lambda):=\left\langle\bar{T} \psi, E_{H}(\lambda) \phi\right\rangle-\left\langle E_{H}(\lambda) \psi, \bar{T} \phi\right\rangle$. Then, by (6.15) and the spectral theorem, we have

$$
\int_{\mathbb{R}} f(\lambda) d \sigma(\lambda)=i \int_{\mathbb{R}} f^{\prime}(\lambda) d \mu(\lambda) .
$$

Applying to the 1.h.s. the integration by parts formula on the Stieltjes integral, we have

$$
-\int_{\mathbb{R}} f^{\prime}(\lambda) \sigma(\lambda) d \lambda=i \int_{\mathbb{R}} f^{\prime}(\lambda) d \mu(\lambda)
$$


It is easy to see that the functional $\Phi: \mathcal{S}(\mathbb{R}) \rightarrow \mathbb{C}$ defined by

$$
\Phi(g):=i \int_{\mathbb{R}} g(\lambda) d \mu(\lambda)+\int_{\mathbb{R}} g(\lambda) \sigma(\lambda) d \lambda, \quad g \in \mathcal{S}(\mathbb{R})
$$

is a tempered distribution on $\mathbb{R}$. Eq.(6.16) implies that $\Phi\left(f^{\prime}\right)=0$ for all $f \in \mathcal{S}(\mathbb{R})$. Hence $\Phi(f)=\alpha \int_{\mathbb{R}} f(\lambda) d \lambda$ with $\alpha$ being a constant. This implies that the measure $\mu$ is absolutely continuous and its Radon-Nikodym derivative $d \mu / d \lambda$ is given by

$$
\frac{d \mu(\lambda)}{d \lambda}=-i \alpha+i \sigma(\lambda)
$$

By a limiting argument, the absolute continuity of $\mu$ can be extended to that of $\left\langle\psi, E_{H}(\cdot) C\right.$ $\times \phi\rangle$ for all $\psi, \phi \in \mathcal{H}$. Hence, in particular, $H \mid \overline{\operatorname{Ran}(C)}$ is absolutely continuous. It follows from (6.17) that, for all $\lambda \in \mathbb{R}$

$$
|\alpha| \leq|\mu(\lambda+1)-\mu(\lambda)|+\sup _{\lambda^{\prime} \in(\lambda, \lambda+1]}\left|\sigma\left(\lambda^{\prime}\right)\right| .
$$

Noting the fact that $\lim _{\lambda \rightarrow-\infty} \mu(\lambda)=0, \lim _{\lambda \rightarrow-\infty} \sigma(\lambda)=0$, we obtain $\alpha=0$. Hence (6.14) follows.

\section{Absence of Minimum-Uncertainty States}

Let $(A, B, C)$ be a triple obeying the GWCCR. A vector $\psi_{0} \in D(A) \cap D(B) \cap(\operatorname{ker} C)^{\perp}$ with $\left\|\psi_{0}\right\|=1$ which attains the equality $(\Delta A)_{\psi_{0}}(\Delta B)_{\psi_{0}}=\delta_{C} / 2>0$ in the uncertainty relation (4.5) with $a=\left\langle\psi_{0}, A \psi_{0}\right\rangle$ and $b=\left\langle\psi_{0}, B \psi_{0}\right\rangle$ is called a minimum-unertainty state for $(A, B, C)$.

Remark 7. 1 It is well-known that the Schrödinger representation $(q, p)$ of the CCR has minimum-uncertainty states. For example, the vector $f_{0} \in L^{2}(\mathbb{R})$ given by $f_{0}(x):=$ $(2 \pi)^{-1 / 4} \sigma^{-1 / 2} e^{-(x-a)^{2} /\left(4 \sigma^{2}\right)}, x \in \mathbb{R}$ with $a \in \mathbb{R}$ and $\sigma>0$ being constants is a minimumuncertainty state for $(q, p):(\Delta q)_{f_{0}}(\Delta p)_{f_{0}}=1 / 2$. It follows from this fact that every representation $(Q, P)$ of the CCR unitarily equivalent to the Schrödinger one has minimumuncertainty states. In particular, by the von Neumann uniqueness theorem mentioned in Introduction of the present paper, every Weyl representation on a separable Hilbert space has minimum-uncertainty states. Also the Fock representation of the CCR with one degree of freedom has minimum-uncertainty states (see, e.g., [10, 11.5.1, 11.5.2]).

In this section, in contrast to the facts stated in Remark 7.1, we give a sufficient condition for a triple $(T, H, C)$ to have no minimum-unertainty states.

Theorem 7. 1 (Absence of minimum-uncertainty state) Suppose that ( $\left.T, H, K_{C}\right)$ obeys the $G W W R$ with $T$ being closed. Assume that $H$ is bounded from below and that $C \geq 0$ with $\delta_{C}>0$. Then there exist no vectors $\psi_{0} \in D(H) \cap D(T) \cap(\operatorname{ker} C)^{\perp}$ with $\left\|\psi_{0}\right\|=1$ such that

$$
(\Delta T)_{\psi_{0}}(\Delta H)_{\psi_{0}}=\frac{\delta_{C}}{2}>0
$$


Remark 7. 2 An essential condition in this theorem is the boundedness below of $H$ (note that the operators $q$ and $p$ in the Schrödinegr representation of the CCR are unbounded both above and below with $\sigma(q)=\sigma(p)=\mathbb{R}$ ).

Remark 7.3 Theorem 7. 1 is an extension of [11, Theorem 5.1], where the case $C=I$ is considered. A new point here is that one does not need to assume the analytic continuation property of the weak Weyl relation (the GWWR with $C=I$ ) as is done in [11, Theorem $5.1]$.

To prove Theorem 7.1, we need two lemmas.

Lemma 7.2 Assume that $(A, B, C)$ obeys the $G W C C R$ with $C \geq 0$. Suppose that there exists a unit vector $\psi \in D(A) \cap D(B) \cap(\operatorname{ker} C)^{\perp}$ such that

$$
\begin{aligned}
& (A+a B+b) \psi=0, \\
& \langle A \psi, B \psi\rangle+\langle B \psi, A \psi\rangle-2\langle\psi, A \psi\rangle\langle\psi, B \psi\rangle=0,
\end{aligned}
$$

where $a$ and $b$ are complex constants. Then $\Re a=0$ and $\Im a>0$.

Proof: In the same way as in the proof of [11, Lemma 5.3], we can show that $i\langle\psi, C \psi\rangle=2 a(\Delta B)_{\psi}^{2}$. By Proposition 4.2, $\psi$ cannot be an eigenvector of $B$. Hence $(\Delta B)_{\psi} \neq 0$. Thus $\Re a=0$ and $\langle\psi, C \psi\rangle=2(\Im a)(\Delta B)_{\psi}^{2}$. The l.h.s. on the second equation is strictly positive, since $C \geq 0$ and $\psi \in(\operatorname{ker} C)^{\perp}$. Hence $\Im a>0$.

For a self-adjoint operator $A$ on a Hilbert space, we define

$$
E_{0}(A):=\inf \sigma(A)
$$

called the ground state energy of $A$, provided that $A$ is bounded from below. If $E_{0}(A)$ is an eigenvalue of $A$, then a non-zero vector in $\operatorname{ker}\left(A-E_{0}(A)\right)$ is called a ground state of $A$.

By the variational principle, one has

$$
E_{0}(A)=\inf _{\psi \in D(A),\|\psi\|=1}\langle\psi, A \psi\rangle .
$$

The following lemma is well known (e.g., [4, Theorem 6.16]).

Lemma 7.3 Let A be a self-adjoint operator on a Hilbert space and bounded from below. Suppose that there exists a unit vector $\psi_{0} \in D(A)$ such that $\left\langle\psi_{0}, A \psi_{0}\right\rangle=E_{0}(A)$. Then $A \psi_{0}=E_{0}(A) \psi_{0}$, i.e., $\psi_{0}$ is a ground state.

\section{Proof of Theorem 7.1}

It is sufficient to prove Theorem 7.1 in the case where $H \geq 0$. Suppose that there existed a unit vector $\psi_{0} \in D(T) \cap D(H) \cap(\operatorname{ker} C)^{\perp}$ satisfying (7.1). Set $\hat{T}:=T-$ $\left\langle\psi_{0}, T \psi_{0}\right\rangle, \hat{H}:=H-\left\langle\psi_{0}, H \psi_{0}\right\rangle$. Then, from the derivation of (4.5)[17, Lemma 1], we have

$$
\begin{aligned}
\left\|\hat{T} \psi_{0}\right\|\left\|\hat{H} \psi_{0}\right\| & =\left|\left\langle\hat{T} \psi_{0}, \hat{H} \psi_{0}\right\rangle\right|=\left|\Im\left\langle\hat{T} \psi_{0}, \hat{H} \psi_{0}\right\rangle\right| \\
& =\frac{1}{2}\left\langle\psi_{0}, C \psi_{0}\right\rangle=\frac{1}{2} \delta_{C} .
\end{aligned}
$$


The first equality is the equality in the Schwarz inequality for $\left|\left\langle\hat{T} \psi_{0}, \hat{H} \psi_{0}\right\rangle\right|$. By this fact and the condition $\delta_{C}>0$, there exists a constant $c \in \mathbb{C}, c \neq 0$ such that

$$
\hat{T} \psi_{0}+c \hat{H} \psi_{0}=0
$$

The second equality in (7.4) implies that $\Re\left\langle\hat{T} \psi_{0}, \hat{H} \psi_{0}\right\rangle=0$. Hence

$$
\left\langle T \psi_{0}, H \psi_{0}\right\rangle+\left\langle H \psi_{0}, T \psi_{0}\right\rangle-2\left\langle\psi_{0}, T \psi_{0}\right\rangle\left\langle\psi_{0}, H \psi_{0}\right\rangle=0
$$

The forth equality in (7.4) and Lemma 7.3 imply that

$$
C \psi_{0}=\delta_{C} \psi_{0}
$$

By (7.5), (7.6) and Lemma 7.2, we have $\Re c=0$ and $\Im c>0$. Hence, let $c=i a$ with $a>0$. Then (7.5) implies that

$$
T \psi_{0}+i a H \psi_{0}+b \psi_{0}=0
$$

where $b$ is a constant. Hence $\left\langle\psi_{0}, T \psi_{0}\right\rangle+i a\left\langle\psi_{0}, H \psi_{0}\right\rangle+b=0$, which implies that $\Im b=$ $-a\left\langle\psi_{0}, H \psi_{0}\right\rangle<0$, since $H \geq 0$ and $H^{1 / 2} \psi_{0} \neq 0$ (see (7.5)). Let $z \in \mathbb{C}$ with $\Re z>0$. Then, by (6.11), (7.8) and (7.7), we have

$$
T e^{-z H} \psi_{0}=e^{-z H}\left(-i a H \psi_{0}-b \psi_{0}\right)-i z \delta_{C} e^{-z H} \psi_{0} .
$$

Since $\Re\left(i b / \delta_{C}\right)>0$, we can take $z=i b / \delta_{C}$ so that $T e^{-z H} \psi_{0}=-i a e^{-z H} H \psi_{0}$. Hence

$$
\left\langle e^{-z H} \psi_{0}, T e^{-z H} \psi_{0}\right\rangle=-i a\left\langle e^{-z H} \psi_{0}, H e^{-z H} \psi_{0}\right\rangle .
$$

The l.h.s. is real and the r.h.s. is pure imaginary. Hence the both sides must vanish. Hence $\left\langle e^{-z H} \psi_{0}, H e^{-z H} \psi_{0}\right\rangle=0$, which implies that $H^{1 / 2} \psi_{0}=0$. But this is a contradiction.

\section{Power Decays of Transition Probability Ampli- tudes in Quantum Dynamics}

In Section 3 we have derived an estimate for transition probability amplitudes in time $t$. In this section we consider a triple $\left(T, H, K_{C}\right)$ obeying the GWWR (discussed in Section 6.2) and show that, for state vectors in "smaller" subspaces, transition probability amplitudes decay in powers of $t$ as $|t| \rightarrow \infty$. We apply the results to two-point correlation functions of Heisenberg operators. We also discuss decays of heat semi-groups $e^{-\beta H}$ on $\beta>0$ the inverse of the absolute temperature.

Let $H$ be a self-adjoint operator on a Hilbert space $\mathcal{H}$ and $C \neq 0$ be a bounded self-adjoint opeartor on $\mathcal{H}$. We introduce a set of generalized time operators:

$$
\mathrm{T}(H, C):=\left\{T \mid\left(T, H, K_{C}\right) \text { obeys the GWWR }\right\} .
$$

By Proposition 2.5, if $T \in \mathrm{T}(H, C)$, then $T+S \in \mathrm{T}(H, C)$ for all symmetric operators $S$ on $\mathcal{H}$ strongly commuting with $H$ such that $D(T) \cap D(S)$ is dense in $\mathcal{H}$. 


\subsection{A simple case}

Theorem 8.1 Let $T \in \mathrm{T}(H, C)$ and $\psi, \phi \in D(T)$. Then, for all $t \in \mathbb{R} \backslash\{0\}$,

$$
\left|\left\langle\phi, e^{-i t H} C \psi\right\rangle\right| \leq \frac{1}{|t|}(\|T \phi\|\|\psi\|+\|\phi\|\|T \psi\|) .
$$

Proof: In the present case, we have $L_{\alpha}=C$ with $\alpha=1$. Hence Proposition 3. 1 gives the desired result.

Remark 8.1 For vectors $\phi, \psi \in \mathcal{H}$, we can define a set of operators

$$
\mathrm{T}_{\phi, \psi}(H, C):=\{T \in \mathrm{T}(H, C) \mid \phi, \psi \in D(T)\}
$$

and put

$$
c_{\phi, \psi}:=\inf _{T \in \mathbf{T}_{\phi, \psi}(H, C)}(\|T \phi\|\|\psi\|+\|\phi\|\|T \psi\|),
$$

then (8.2) implies that

$$
\left|\left\langle\phi, e^{-i t H} C \psi\right\rangle\right| \leq \frac{c_{\phi, \psi}}{|t|} .
$$

Remark 8. 2 Let $T \in \mathrm{T}(H, C)$. Then, for all $\psi \in D(T)$ with $\|\psi\|=1, T-\langle\psi, T \psi\rangle$ is in the set $\mathrm{T}(H, C)$. Hence (8.2) implies that

$$
\left|\left\langle\psi, e^{-i t H} C \psi\right\rangle\right|^{2} \leq \frac{4(\Delta T)_{\psi}^{2}}{t^{2}}
$$

Hence Theorem 8. 1 gives a generalization of [11, Theorem 4.1].

\subsection{Higher order dcays in smaller subspaces}

As demonstrated in a concrete example [11, Proposition 3.2], the modulus of a transition probability amplitude $\left|\left\langle\phi, e^{-i t H} \psi\right\rangle\right|$ may decay faster than $|t|^{-1}$ as $|t| \rightarrow \infty$ for a class of vectors $\phi$ and $\psi$. In this subsection we investigate this aspect in an abstract framework and show that $\left|\left\langle\phi, e^{-i t H} \psi\right\rangle\right|$ decays faster than $|t|^{-1}$ for all $\phi$ and $\psi$ in smaller subspaces.

Lemma 8.2 Let $T \in \mathrm{T}(H, C)$. Suppose that

$$
C T \subset T C \text {. }
$$

Then, for all $n \in \mathbb{N}$ and $t \in \mathbb{R}$,

$$
\begin{gathered}
e^{-i t H} D\left(T^{n}\right)=D\left(T^{n}\right) \\
T^{n} e^{-i t H}=e^{-i t H}(T+t C)^{n} .
\end{gathered}
$$


Proof: By (2.8) we have the operator equality $e^{i t H} T^{n} e^{-i t H}=(T+t C)^{n}$. Condition (8.5) implies that $D\left((T+t C)^{n}\right)=D\left(T^{n}\right)$. Hence 8.6) follows and (8.7) holds.

Theorem 8. 3 Let $T \in \mathrm{T}(H, C)$. Assume (8.5). Let $n \in \mathbb{N}$ and $\psi, \phi \in D\left(T^{n}\right)$. We define constants $d_{k}^{T}(\phi, \psi), k=1, \cdots, n$ by the following recursion relation:

$$
\begin{aligned}
d_{1}^{T}(\phi, \psi) & :=\|T \phi\|\|\psi\|+\|\phi\|\|T \psi\|, \\
d_{n}^{T}(\phi, \psi) & :=\left\|T^{n} \phi\right\|\|\psi\|+\|\phi\|\left\|T^{n} \psi\right\|+\sum_{r=1}^{n-1}{ }_{n} C_{r} d_{n-r}^{T}\left(\phi, T^{r} \psi\right), n \geq 2,
\end{aligned}
$$

where ${ }_{n} C_{r}:=n ! /[(n-r) ! r !]$. Then, for all $t \in \mathbb{R} \backslash\{0\}$,

$$
\left|\left\langle\phi, e^{-i t H} C^{n} \psi\right\rangle\right| \leq \frac{d_{n}^{T}(\phi, \psi)}{|t|^{n}}
$$

Proof: We prove (8.10) by induction in $n$. The case $n=1$ holds by Theorem 8. 1 . Suppose that (8.10) holds for $n=1, \cdots, m-1(m \geq 2)$. Let $\psi, \phi \in D\left(T^{m}\right)$. Condition (8.5) implies that, for all $k, r \in \mathbb{N}, C^{k} T^{r} \subset T^{r} C^{k}$. By this fact and Lemma 8. 2, we have

$$
\begin{aligned}
T^{m} e^{-i t H} \psi & =e^{-i t H}(T+t C)^{m} \psi \\
& =e^{-i t H} T^{m} \psi+\sum_{r=1}^{m-1}{ }_{m} C_{r} t^{m-r} e^{-i t H} C^{m-r} T^{r} \psi+t^{m} e^{-i t H} C^{m} \psi
\end{aligned}
$$

Hence

$$
\begin{aligned}
|t|^{m}\left|\left\langle\phi, e^{-i t H} C^{m} \psi\right\rangle\right| \leq & \left\|T^{m} \phi\right\|\|\psi\|+\|\phi\|\left\|T^{m} \psi\right\| \\
& +\sum_{r=1}^{m-1}{ }_{m} C_{r}|t|^{m-r}\left|\left\langle\phi, e^{-i t H} C^{m-r} T^{r} \psi\right\rangle\right| .
\end{aligned}
$$

By the induction hypothesis, we have

$$
|t|^{m-r}\left|\left\langle\phi, e^{-i t H} C^{m-r} T^{r} \psi\right\rangle\right| \leq d_{m-r}^{T}\left(\phi, T^{r} \psi\right) .
$$

Hence (8.10) with $n=m$ follows.

Theorem 8.3 can be generalized. We need a lemma.

Lemma 8. 4 Let $T_{1}, \cdots, T_{n} \in \mathrm{T}(H, C)(n \in \mathbb{N})$ such that $C T_{j} \subset T_{j} C, j=1, \cdots, n$. Then, for all $t \in \mathbb{R}$,

$$
e^{-i t H} D\left(\left(T_{1}+t C\right) \cdots\left(T_{n}+t C\right)\right) \subset D\left(T_{1} \cdots T_{n}\right)
$$

and, for all $\psi \in D\left(\left(T_{1}+t C\right) \cdots\left(T_{n}+t C\right)\right)$,

$$
T_{1} \cdots T_{n} e^{-i t H} \psi=e^{-i t H}\left(T_{1}+t C\right) \cdots\left(T_{n}+t C\right) \psi .
$$


Proof: We prove the assertion by induction in $n$. The case $n=1$ obviously holds. Suppose that, for an $m \in \mathbb{N},(8.11)$ and (8.12) hold. Let $\phi \in D\left(\left(T_{1}+t C\right) \cdots\left(T_{m+1}+t C\right)\right)$. Then the vector $\left(T_{m+1}+t C\right) \phi$ is in $D\left(\left(T_{1}+t C\right) \cdots\left(T_{m}+t C\right)\right)$. Hence, by the induction hypothesis, $e^{-i t H}\left(T_{m+1}+t C\right) \phi$ is in $D\left(T_{1} \cdots T_{m}\right)$ and

$$
T_{1} \cdots T_{m} e^{-i t H}\left(T_{m+1}+t C\right) \phi=e^{-i t H}\left(T_{1}+t C\right) \cdots\left(T_{m+1}+t C\right) \phi .
$$

On the other hand, $e^{-i t H} \phi$ is in $D\left(T_{m+1}\right)$ and $T_{m+1} e^{-i t H} \phi=e^{-i t H}\left(T_{m+1}+t C\right) \phi$. Hence $T_{m+1} e^{-i t H} \phi$ is in $D\left(T_{1} \cdots T_{m}\right)$, i.e., $e^{-i t H} \phi \in D\left(T_{1} \cdots T_{m+1}\right)$, and (8.12) with $n=m+1$ holds. Thus the assertion holds for $n=m+1$.

Theorem 8. 5 Let $T, T_{1}, \cdots, T_{n} \in \mathrm{T}(H, C)$ such that $C T \subset T C, C T_{j} \subset T_{j} C, j=$ $1, \cdots, n$. Let $\phi \in D\left(T_{n} \cdots T_{1}\right) \cap D\left(T^{n-1}\right)$ and $\psi \in \cap_{r=1}^{n-1} \cap_{1 \leq i_{1}<\cdots<i_{r} \leq n} D\left(T^{n-r} T_{i_{1}} \cdots T_{i_{r}}\right)$. For $k=1, \cdots, n$, we define a constant

$$
\begin{aligned}
\delta_{n}^{T}\left(\phi, \psi ; T_{1}, \cdots, T_{n}\right):= & \left\|T_{n} \cdots T_{1} \phi\right\|\|\psi\|+\|\phi\|\left\|T_{1} \cdots T_{n} \psi\right\| \\
& +\sum_{r=1}^{n-1} \sum_{1 \leq i_{1}<\cdots<i_{r} \leq n} d_{n-r}^{T}\left(\phi, T_{i_{1}} \cdots T_{i_{r}} \psi\right) .
\end{aligned}
$$

Then, for all $t \in \mathbb{R} \backslash\{0\}$,

$$
\left|\left\langle\phi, e^{-i t H} C^{n} \psi\right\rangle\right| \leq \frac{\delta_{n}^{T}\left(\phi, \psi ; T_{1}, \cdots, T_{n}\right)}{|t|^{n}} .
$$

Proof: By Lemma 8.4, we have

$$
\begin{aligned}
T_{1} \cdots T_{n} e^{-i t H} \psi= & e^{-i t H} T_{1} \cdots T_{n} \psi \\
& +\sum_{r=1}^{n-1} t^{n-r} \sum_{1 \leq i_{1}<\cdots<i_{r} \leq n} e^{-i t H} C^{n-r} T_{i_{1}} \cdots T_{i_{r}} \psi+t^{n} e^{-i t H} C^{n} \psi .
\end{aligned}
$$

Hence

$$
\begin{aligned}
|t|^{n}\left|\left\langle\phi, e^{-i t H} C^{n} \psi\right\rangle\right| \leq & \left\|T_{n} \cdots T_{1} \phi\right\|\|\psi\|+\|\phi\|\left\|T_{1} \cdots T_{n} \psi\right\| \\
& +\sum_{r=1}^{n-1}|t|^{n-r} \sum_{1 \leq i_{1}<\cdots<i_{r} \leq n} \mid\left\langle\phi, e^{-i t H} C^{n-r} T_{i_{1}} \cdots T_{i_{r}} \psi\right\rangle
\end{aligned}
$$

Note that $\phi, T_{i_{1}} \cdots T_{i r} \psi \in D\left(T^{n-r}\right)$ for $r=1, \cdots, n-1$. Hence, by Theorem 8.3 , we have

$$
|t|^{n-r}\left|\left\langle\phi, e^{-i t H} C^{n-r} T_{i_{1}} \cdots T_{i_{r}} \psi\right\rangle\right| \leq d_{n-r}^{T}\left(\phi, T_{i_{1}} \cdots T_{i_{r}} \psi\right)
$$

Thus (8.15) follows.

Finally we discuss the case where condition (8.5) is not necessarily satisfied. For $n \geq 2$ and $r=1, \cdots, n-1$, we introduce a set

$$
\mathrm{J}_{n, r}:=\left\{j:=\left(j_{1}, \cdots, j_{r+1}\right) \in\{0,1\}^{r+1} \mid j_{1}+\cdots+j_{r+1}=n-r\right\}
$$


and, for each $j \in \mathrm{J}_{n, r}$, we define

$$
K_{n, r}^{(j)}:=T^{j_{1}} C T^{j_{2}} C \cdots C T^{j_{r}} C T^{j_{r+1}} .
$$

Let

$$
\begin{aligned}
& \mathcal{D}_{n}(T, C) \\
& :=\left\{\psi \in D\left(T^{n}\right) \cap\left(\cap_{r=1}^{n-1} \cap_{j \in \mathrm{J}_{n, r}} D\left(K_{n, r}^{(j)}\right)\right) \mid K_{n, r}^{(j)} \psi \in \operatorname{Ran}\left(C^{r} \mid D\left(T^{r}\right)\right),\right. \\
& \left.j \in \mathrm{J}_{n, r}, r=1, \cdots, n-1\right\} \quad(n \geq 2) .
\end{aligned}
$$

We set $\mathcal{D}_{1}(T, C):=D(T)$.

Remark 8. 3 If (8.5) holds, then $\mathcal{D}_{n}(T, C)=D\left(T^{n}\right)$.

Theorem 8. 6 Let $T \in \mathrm{T}(H, C)$. Then, for all $\phi \in D\left(T^{n}\right)$ and $\psi \in \mathcal{D}_{n}(T, C)$ and $t \in \mathbb{R} \backslash\{0\}$,

$$
\left|\left\langle\phi, e^{-i t H} C^{n} \psi\right\rangle\right| \leq \frac{d_{n}(\phi, \psi)}{|t|^{n}},
$$

where $d_{n}(\phi, \psi)>0$ is a constant independent of $t$.

Proof: We prove (8.19) by induction in $n$. The case $n=1$ is just Theorem 8. 1 . Suppose that (8.19) holds with $n=1, \cdots, m-1(m \geq 2)$. For all $\psi \in \mathcal{D}_{m}(T, C)$ we have

$$
T^{m} e^{-i t H} \psi=e^{-i t H} T^{m} \psi+t^{m} e^{-i t H} C^{m} \psi+\sum_{r=1}^{m-1} t^{r} \sum_{j \in J_{m, r}} e^{-i t H} K_{m, r}^{(j)} \psi .
$$

Hence, for all $\phi \in D\left(T^{m}\right)$,

$$
\begin{aligned}
|t|^{m}\left\langle\phi, e^{-i t H} C^{m} \psi\right\rangle \leq & \left\|T^{m} \phi\right\|\|\psi\|+\|\phi\|\left\|T^{m} \psi\right\| \\
& +\sum_{r=1}^{m-1}|t|^{r} \sum_{j \in J_{m, r}}\left|\left\langle\phi, e^{-i t H} K_{m, r}^{(j)} \psi\right\rangle\right| .
\end{aligned}
$$

Since $K_{m, r}^{(j)} \psi \in \operatorname{Ran}\left(C^{r} \mid D\left(T^{r}\right)\right)$, there is a vector $\chi_{j} \in D\left(T^{r}\right)$ such that $K_{m, r}^{(j)} \psi=C^{r} \chi_{j}$. By this fact and the induction hypothesis, we have $|t|^{r}\left|\left\langle\phi, e^{-i t H} K_{m, r}^{(j)} \psi\right\rangle\right| \leq c_{m, r}$ with a constant $c_{m, r}$ independet of $t$. Thus (8.19) with $n=m$ follows.

\subsection{Correlation functions}

In this subsection, we show that the existence of generalized time-operators gives upper bounds for correlation functions for a class of linear operators. For a linear operator $A$ on $\mathcal{H}$ and a self-adjoint operator $H$ on $\mathcal{H}$, we define

$$
A(t):=e^{i t H} A e^{-i t H}, \quad t \in \mathbb{R},
$$


the Heisenberg operator of $A$ with respect to $H$. Let $B$ be a linear operator on $\mathcal{H}$. Let

$$
\psi \in \cap_{t \in \mathbb{R}}\left[D\left(A e^{-i t H}\right) \cap D\left(B e^{-i t H}\right)\right]
$$

with $\|\psi\|=1$. Then we can define

$$
W(t, s ; \psi):=\langle A(t) \psi, B(s) \psi\rangle, \quad s, t \in \mathbb{R} .
$$

We call it the two-point correlation function of $A$ and $B$ with repect to the vector $\psi$.

Theorem 8.7 Let $T \in \mathrm{T}(H, C)$. Suppose that $\psi$ is an eigenvector of $H$ such that $A \psi \in D(T)$ and $B \psi \in \operatorname{Ran}(C \mid D(T))$. Then, for all $t, s \in \mathbb{R}$ with $t \neq s$,

$$
|W(t, s ; \psi)| \leq \frac{c_{A, B, T}}{|t-s|}
$$

where

$$
c_{A, B, T}:=\inf _{\chi \in D(T), B \psi=C \chi}\|T A \psi\|\|\chi\|+\|A \psi\|\|T \chi\| .
$$

Proof: Let $E$ be the eigenvalue of $H$ with eigenvector $\psi$. Then we have

$$
W(t, s)=e^{i(t-s) E}\left\langle A \psi, e^{-i(t-s) H} B \psi\right\rangle .
$$

There exists a vector $\chi \in D(T)$ such that $B \psi=C \chi$. Hence, applying Theorem 8. 1, we obtain

$$
|W(t, s ; \psi)| \leq \frac{\|T A \psi\|\|\chi\|+\|A \psi\|\|T \chi\|}{|t-s|} .
$$

Thus (8.22) follows.

Theorem 8.7 can be strengthened:

Theorem 8. 8 Let $T \in \mathrm{T}(H, C)$ with (8.5). Suppose that $\psi$ is an eigenvector of $H$ such that $\psi \in D(A)$ and $A \psi \in D\left(T^{n}\right)$ and $B \psi \in \operatorname{Ran}\left(C^{n} \mid D\left(T^{n}\right)\right)$. Then, for all $t, s \in \mathbb{R}$ with $t \neq s$,

$$
|W(t, s)| \leq \frac{c_{A, B, T}^{(n)}}{|t-s|^{n}},
$$

where

$$
c_{A, B, T}^{(n)}:=\inf _{\chi \in D\left(T^{n}\right), B \psi=C^{n} \chi} d_{n}^{T}(A \psi, \chi)
$$

Proof: This follow from (8.23) and an application of Theorem 8. 3 . 


\subsection{Heat semi-groups}

In this subsection we assume the following:

Hypothesis $(\mathbf{H})$ The self-adjoint operator $H$ is bounded from below and there exists a closed symmetric operator $T$ such that $T \in \mathrm{T}(H, C)$.

Then (6.11) holds. We set

$$
\hat{H}:=H-E_{0}(H) .
$$

We have for all $\beta \geq 0$

$$
\left\|e^{-\beta \hat{H}}\right\|=1 \text {. }
$$

Hence, in the same way as in Subsections 8.1 and 8.2, we obtain the following results on the decay (in $\beta$ ) of the quantity $\left\langle\phi, e^{-\beta \hat{H}} C \psi\right\rangle$.

Theorem 8.9 Let $\phi, \psi \in D(T)$. Then, for all $\beta>0$,

$$
\left|\left\langle\phi, e^{-\beta \hat{H}} C \psi\right\rangle\right| \leq \frac{1}{\beta}(\|T \phi\|\|\psi\|+\|\phi\|\|T \psi\|) .
$$

Theorem 8.10 Assume (8.5). Then, for all $\phi, \psi \in D\left(T^{n}\right)$ and $\beta>0$,

$$
\left|\left\langle\phi, e^{-\beta \hat{H}} C^{n} \psi\right\rangle\right| \leq \frac{d_{n}^{T}(\phi, \psi)}{\beta^{n}}
$$

Theorem 8.11 For all $\phi \in D\left(T^{n}\right), \psi \in \mathcal{D}_{n}(T, C)$ and $\beta>0$,

$$
\left|\left\langle\phi, e^{-\beta \hat{H}} C^{n} \psi\right\rangle\right| \leq \frac{c_{n}}{\beta^{n}},
$$

where $c_{n}$ is a constant independent of $\beta$.

\section{Abstract Version of Wigner's Time-Energy Un- certainty Relation}

In this section we apply Theorems 8.1 and 8. 6 to establish an abstract version of Wigner's time-energy uncertainty relation [23].

Let $H$ be a self-adjoint operator on a Hilbert space $\mathcal{H}$. In the context of quantm mechanics where $H$ is interpreted as the Hamiltonian of a quantum system, the state vector at time $t \in \mathbb{R}$ is given by

$$
\psi(t):=e^{-i t H} \psi
$$

with $\psi \in \mathcal{H}$ being the initial state. Let $\phi_{0} \in \mathcal{H}$ and suppose that

$$
\int_{\mathbb{R}} t^{2}\left|\left\langle\phi_{0}, \psi(t)\right\rangle\right|^{2} d t<\infty .
$$

Then

$$
N_{0}:=\int_{\mathbb{R}}\left|\left\langle\phi_{0}, \psi(t)\right\rangle\right|^{2} d t
$$


is finite and we can define

$$
\begin{aligned}
\langle t\rangle & :=\frac{\int_{\mathbb{R}} t\left|\left\langle\phi_{0}, \psi(t)\right\rangle\right|^{2} d t}{N_{0}} \\
\Delta t & :=\left(\frac{\int_{\mathbb{R}}(t-\langle t\rangle)^{2}\left|\left\langle\phi_{0}, \psi(t)\right\rangle\right|^{2} d t}{N_{0}}\right)^{1 / 2} .
\end{aligned}
$$

Physically $\langle t\rangle$ may be interpreted as the expectation value of the "arrival time", i.e., the time $t$ at when the state $\psi(t)$ "arrives" at $\phi_{0}$. In this interpretation, $\Delta t$ expresses the standard deviation of the arrival time of the intial state $\psi$ to the state $\phi_{0}$.

We define

$$
\left.f_{H}\left(t ; \phi_{0}, \psi\right)\right):=\left\{\begin{array}{ll}
\left\langle\phi_{0}, H \psi(t)\right\rangle & ; \text { if } \psi \in D(H) \\
\left\langle H \phi_{0}, \psi(t)\right\rangle & ; \text { if } \phi_{0} \in D(H)
\end{array} .\right.
$$

We assume that $\phi_{0} \in D(H)$ or $\psi \in D(H)$ and

$$
\int_{\mathbb{R}}\left|f_{H}\left(t ; \phi_{0}, \psi\right)\right|^{2} d t<\infty
$$

Then we can define

$$
\begin{aligned}
\left\langle\mathcal{E}_{H}\right\rangle & :=\frac{\int_{\mathbb{R}}\left\langle\psi(t), \phi_{0}\right\rangle f_{H}\left(t ; \phi_{0}, \psi\right) d t}{N_{0}} \\
\Delta \mathcal{E}_{H} & :=\left(\frac{\int_{\mathbb{R}}\left|f_{H}\left(t ; \phi_{0}, \psi\right)\right|^{2} d t}{N_{0}}-\left\langle\mathcal{E}_{H}\right\rangle^{2}\right)^{1 / 2} .
\end{aligned}
$$

Theorem 9. 1 Suppose that $\phi_{0} \in D(H)$ or $\psi \in D(H)$ and that (9.2) and (9.7) hold. Then

$$
\Delta t \cdot \Delta \mathcal{E}_{H} \geq \frac{1}{2}
$$

Proof : From the Schrödinger representation $(\widehat{E}, \widehat{t})$ of the CCR in $L^{2}(\mathbb{R})$, where $\widehat{t}$ is the multiplication operator by the coordinate function $t \in \mathbb{R}$ and $\widehat{E}:=i D_{t}$ with $D_{t}$ being the generalized differential operator in the variable $t$, we have the standard uncertainty relation

$$
\|(\widehat{E}-\langle u, \widehat{E} u\rangle) u\| \cdot\|(\widehat{t}-\langle u, \widehat{t} u\rangle) u\| \geq \frac{1}{2}
$$

for all $u \in D(\widehat{E}) \cap D(\widehat{t})$ with $\|u\|=1$. We define $f: \mathbb{R} \rightarrow \mathbb{C}$ by $f(t):=\left\langle\phi_{0}, \psi(t)\right\rangle$. Then, by $(9.2)$ and (9.7), $f$ is in $D(\widehat{t}) \cap D(\widehat{E})$ with $\widehat{E} f(t)=f_{H}\left(t ; \phi_{0}, \psi\right)$. Let $\widetilde{f}(t):=f(t) /\|f\|$. Then it is easy to see that

$$
\Delta \mathcal{E}_{H}=\|(\widehat{E}-\langle\tilde{f}, \widehat{E} \tilde{f}\rangle) \tilde{f}\|, \quad \Delta t:=\|(\widehat{t}-\langle\tilde{f}, \widehat{t} \tilde{f}\rangle) \tilde{f}\| .
$$

Hence (9.10) follows.

Theorem 9. 2 Let $T \in \mathrm{T}(H, C)$. Suppose that $\psi=C^{2} \chi$ with $\chi \in D\left(T^{2}\right) \cap D(T C)$ satisfying $T C \chi \in \operatorname{Ran}(C \mid D(T))$ and that $\phi_{0} \in D\left(T^{2}\right) \cap D(T H)$. Then (9.10) holds. 
Proof: By the present assumption, $\phi_{0}$ is in $D(H)$ with $H \phi_{0} \in D(T)$ and $\psi=C(C \chi)$ with $C \chi \in D(T)$. Hence we can apply Theorem 8.1 to obtain

$$
\left|\left\langle H \phi_{0}, \psi(t)\right\rangle\right| \leq \frac{1}{|t|}\left(\left\|T H \phi_{0}\right\|\|C \chi\|+\left\|H \phi_{0}\right\|\|T C \chi\|\right) .
$$

Using this estimate, we see that (9.7) holds. We have $\phi_{0} \in D\left(T^{2}\right)$ and $\chi \in \mathcal{D}_{2}(T, C)$, where $\mathcal{D}_{n}(T, C)$ is defined by (8.18). Hence, by Theorem 8.6, $\left|\left\langle\phi_{0}, \psi(t)\right\rangle\right| \leq c /|t|^{2}$ with $c>0$ a constant. This implies that (9.2) holds. Thus, by Theorem 9.1 , we obtain the desired result.

\section{Structure producing successively triples obeying the GWWR}

Let $\left(Q, P, K_{C}\right)$ be a triple obeying the GWWR in a Hilbert space $\mathcal{H}$, i.e.,

$$
Q e^{-i t P}=e^{-i t P}(Q+t C), \quad \forall t \in \mathbb{R} .
$$

(See Proposition 2.1.)

Lemma 10. 1

(i) For all $\psi \in D(\bar{Q})$ and all $z \in \mathbb{C}$ with $\Re z>0$, we have $e^{-z P^{2}} \psi \in D(\bar{Q})$ and

$$
\bar{Q} e^{-z P^{2}} \psi=e^{-z P^{2}} \bar{Q} \psi-2 i z P e^{-z P^{2}} C \psi .
$$

(ii) For all $\psi \in D(\bar{Q}) \cap D(P)$ and all $t \in \mathbb{R}$, we have $e^{-i t P^{2}} \psi \in D(\bar{Q}) \cap D(P)$ and

$$
\bar{Q} e^{-i t P^{2}} \psi=e^{-i t P^{2}}(\bar{Q}+2 t P C) \psi \text {. }
$$

Proof : (i) Let $\Re z>0$. Then the function : $t \mapsto f(t):=e^{-z t^{2}}, t \in \mathbb{R}$ is in the class $C_{\mathrm{b}}^{1}(\mathbb{R})$ and $f^{\prime}(t)=-2 z t e^{-z t^{2}}$. Hence, applying Theorem 6.2 -(i), we conclude that $e^{-z P^{2}} D(\bar{Q}) \subset D(\bar{Q})$ and $(10.2)$ holds for all $\psi \in D(\bar{Q})$.

(ii) Let $\varepsilon>0$. By part (i), we have for all $\phi \in D\left(Q^{*}\right)=D\left((\bar{Q})^{*}\right)$ and $\psi \in D(\bar{Q}) \cap D(P)$

$$
\left\langle Q^{*} \phi, e^{-(\varepsilon+i t) P^{2}} \psi\right\rangle=\left\langle\phi, e^{-(\varepsilon+i t) P^{2}} \bar{Q} \psi\right\rangle-\left\langle\phi, 2 i(\varepsilon+i t) P e^{-(\varepsilon+i t) P^{2}} C \psi\right\rangle .
$$

Applying Proposition 6.3 with $H=P$ and $T=Q$, we see that $C \psi \in D(P)$. Hence, taking $\varepsilon \rightarrow 0$, we obtain

$$
\left\langle Q^{*} \phi, e^{-i t P^{2}} \psi\right\rangle=\left\langle\phi, e^{-i t P^{2}} \bar{Q} \psi\right\rangle+\left\langle\phi, 2 t P e^{-i t P^{2}} C \psi\right\rangle .
$$

This implies that $e^{-i t P^{2}} \psi \in D\left(Q^{* *}\right)=D(\bar{Q})$ and (10.3) holds.

In the rest of this section, we consider a simple case such that

$$
\operatorname{ker} C=\{0\} .
$$


Then, by Corollary 3. 3 applied to $H=P, T=Q$ and $L_{\alpha}=C$, we have $\sigma_{\mathrm{p}}(P)=\emptyset$. In particular, $P$ is injective. Hence we can define

$$
T(Q, P):=\frac{1}{4}\left[\bar{Q} P^{-1}+\left(\bar{Q} P^{-1}\right)^{*}\right]
$$

provided that $D\left(\bar{Q} P^{-1}\right)$ is dense in $\mathcal{H}$.

Theorem 10.2 Assume (10.4) and that $D\left(\bar{Q} P^{-1}\right)$ is dense in $\mathcal{H}$. Then, for all $t \in \mathbb{R}$, $e^{-i t P^{2}} D(T(Q, P)) \subset D(T(Q, P))$ and

$$
T(Q, P) e^{-i t P^{2}} \psi=e^{-i t P^{2}}(T(Q, P)+t C) \psi, \quad \psi \in D(T(Q, P)) .
$$

Proof: Let $\psi \in D\left(\bar{Q} P^{-1}\right)$. Then $P^{-1} \psi \in D(\bar{Q}) \cap D(P)$. Hence, by Lemma 10. 1 -(ii),

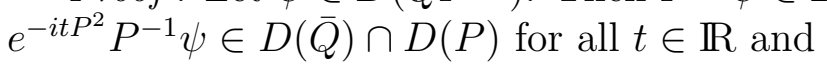

$$
\bar{Q} P^{-1} e^{-i t P^{2}} \psi=e^{-i t P^{2}}\left(\bar{Q} P^{-1}+2 t C\right) \psi,
$$

which implies that, for all $t \in \mathbb{R}, e^{-i t P^{2}}\left(\bar{Q} P^{-1}+2 t C\right) \subset \bar{Q} P^{-1} e^{-i t P^{2}}$. Hence

$$
\left.e^{i t P^{2}}\left(\bar{Q} P^{-1}\right)^{*} \subset\left[\left(\bar{Q} P^{-1}\right)^{*}+2 t C\right) e^{i t P^{2}}\right]
$$

for all $t \in \mathbb{R}$. This implies that $e^{-i t P^{2}}\left[\left(\bar{Q} P^{-1}\right)^{*}+2 t C\right] \subset\left(\bar{Q} P^{-1}\right)^{*} e^{-i t P^{2}}$ for all $t \in \mathbb{R}$. Thus the desired result follows.

Theorem 10. 2 shows that, if $D\left(\bar{Q} P^{-1}\right) \cap D\left(\left(\bar{Q} P^{-1}\right)^{*}\right)$ is dense in addition to the assumption there, then $\left(T(Q, P), P^{2}, K_{C}\right)$ obeys the GWWR. This structure is very interesting, since, apart from domain problems, it produces a series of triples obeying the GWWR. Indeed, let

$$
\begin{aligned}
T_{1}(Q, P) & :=T(Q, P), \\
T_{n}(Q, P) & :=T\left(T_{n-1}(Q, P), P^{2^{n-1}}\right), \quad n \geq 2 .
\end{aligned}
$$

Then $\left(T_{n}(Q, P), P^{2^{n}}, K_{C}\right)$ obeys the GWWR for all $n=1, \cdots, N(N \in \mathbb{N})$, provided that each $n=1, \cdots, N, T_{n}(Q, P)$ is symmetric.

Example 10. 1 A simple example of $(Q, P)$ is given by the Schrödinger representation $(q, p)$ on $L^{2}(\mathbb{R})$ of the CCR with one degree of freedom. It is easy to see that $\left(q, p, K_{I}\right)$ obeys the GWWR. The operator $T(q, p)$ in this case is called the Aharonov-Bohm time operator $[2,11,12]$. Theorem 10. 2 clarifies a general mathematical structure behind this operator. A simple application of Theorem 10. 2 to this special case produces generalized time operartors to $H=(-\Delta)^{2^{n-1}}(n \in \mathbb{N})$.

We can extend the theory presented above to the case of finitely many degrees of freedom. Let $Q_{j}(j=1, \cdots, n, n \in \mathbb{N})$ be a symmetric operator on $\mathcal{H}$ and $P_{j}$ be a selfadjoint operator on $\mathcal{H}$ such that $P_{j}$ strongly commutes with $Q_{k}$ and $P_{k}(j, k=1, \cdots, n, j \neq$ 
$k)$. Suppose that each $\left(Q_{j}, P_{j}, K_{C}\right)$ obeys the GWWR with $C$ satisfying (10.4). Then, as already shown, each $P_{j}$ is injective. Suppose that $D\left(\bar{Q}_{j} P_{j}^{-1}\right)$ is dense. Then we can define

$$
T_{j}:=\frac{1}{4}\left[\bar{Q}_{j} P_{j}^{-1}+\left(\bar{Q}_{j} P_{j}^{-1}\right)^{*}\right], \quad j=1, \cdots, n .
$$

By the strong commutativity of $P_{j}^{\prime}$ 's $(j=1, \cdots, n)$, the operator

$$
H:=\sum_{j=1}^{n} P_{j}^{2}
$$

is self-adjoint and nonnegative.

Theorem 10.3 Let the assumption stated above on $\left(Q_{j}, P_{j}\right)(j=1, \cdots, n)$ be satisfied. Then, for all $t \in \mathbb{R}$ and $\left.j=1, \cdots, n, e^{-i t H} D\left(T_{j}\right)\right) \subset D\left(T_{j}\right)$ and

$$
T_{j} e^{-i t H} \psi=e^{-i t H}\left(T_{j}+t C\right) \psi, \quad \psi \in D\left(T_{j}\right) .
$$

Proof: By the strong commutativity of $Q_{j}$ with $P_{k}(k \neq j)$, we have $Q_{j} e^{-i t H}=$ $e^{-i t H_{j}} Q_{j} e^{-i t P_{j}^{2}}$ on $D\left(P_{j}\right)$, where $H_{j}:=\sum_{k \neq j} P_{k}^{2}$. Hence, applying Theorem 10.2 with $Q=Q_{j}, P=P_{j}$ we obtain the desired result.

In this case too, a remark similar to the one after Theorem 10. 2 is applicable.

\section{Generalized Time Operators of Partial Differen- tial Operators}

In this section we construct classes of generalized time operators of partial differential operators.

\subsection{Constructions from the Schrödinger representation of the CCR with $d$ degrees of freedom}

Let $\mathbf{q}=\left(q_{1}, \cdots, q_{d}\right)$ and $\mathbf{p}=\left(p_{1}, \cdots, p_{d}\right)$ be the Schrödinger representation of the CCR with $d$ degrees of freedom. Namely, $q_{j}$ is the multiplication operator by $x_{j}$, the $j$-th coordinate function $\left(x=\left(x_{1}, \cdots, x_{d}\right) \in \mathbb{R}^{d}\right)$ acting in $L^{2}\left(\mathbb{R}^{d}\right)$ and $p_{j}=-i D_{j}$ on $L^{2}\left(\mathbb{R}^{d}\right)$ $\left(D_{j}\right.$ is the generalized partial differential operator in $x_{j}$ ). The following properties are well known:

(i) $p_{j}$ and $p_{l}$ are strongly commuting self-adjoint operators.

(ii) For all $t \in \mathbb{R}$,

$$
q_{j} e^{-i t p_{l}}=e^{-i t p_{l}}\left(q_{j}+\delta_{j l} t\right), \quad j, l=1, \cdots, d .
$$


We denote by

$$
\hat{\mathbb{R}}^{d}:=\left\{k=\left(k_{1}, \cdots, k_{d}\right) \mid k_{j} \in \mathbb{R}, j=1, \cdots, d\right\}
$$

the $d$-dimensional momentum space and by $C^{r}\left(\hat{\mathbb{R}}^{d}\right)$ the set of $r$ times continuously differentiable functions on $\hat{\mathbb{R}}^{d}$

Let $F \in C^{1}\left(\hat{\mathbb{R}}^{d}\right)$ be real-valued. Then the operators

$$
H_{F}:=F(\mathbf{p})
$$

and

$$
F_{j}^{(1)}:=\left(\partial_{j} F\right)(\mathbf{p})
$$

are self-adjoint. Let $N_{F}^{(j)}$ be a closed subset of $\hat{\mathbb{R}}^{d}$ with Lebesgue measure zero and $G \in C^{1}\left(\hat{\mathbb{R}}^{d} \backslash N_{F}^{(j)}\right)$ such that

$$
\sup _{k \in \hat{\mathbb{R}}^{d} \backslash N_{F}^{(j)}}\left|G(k) \partial_{j} F(k)\right|<\infty
$$

Then we can define a linear operator $T_{F, G}^{(j)}$ on $L^{2}\left(\mathbb{R}^{d}\right)$ by

$$
T_{F, G}^{(j)}:=G(\mathbf{p}) q_{j}+q_{j} G(\mathbf{p})^{*},
$$

with domain

$$
D\left(T_{F, G}^{(j)}\right):=\mathcal{F}^{-1} C_{0}^{1}\left(\hat{\mathbb{R}}^{d} \backslash N_{F}^{(j)}\right),
$$

where $\mathcal{F}: L^{2}\left(\mathbb{R}^{d}\right) \rightarrow L^{2}\left(\hat{\mathbb{R}}^{d}\right)$ is the Fourier transform:

$$
(\mathcal{F} \psi)(k):=\frac{1}{(2 \pi)^{d / 2}} \int_{\mathbb{R}^{d}} e^{-i k x} \psi(x) d x, \quad \psi \in L^{2}\left(\mathbb{R}^{d}\right)
$$

in the $L^{2}$-sense. Note that, for all $u \in C_{0}^{1}\left(\hat{\mathbb{R}}^{d} \backslash N_{F}^{(j)}\right)$,

$$
\left(\mathcal{F} T_{F, G}^{(j)} \mathcal{F}^{-1} u\right)(k)=G(k) i \frac{\partial u(k)}{\partial k_{j}}+i \frac{\partial}{\partial k_{j}}\left[G(k)^{*} u(k)\right], \quad \text { a.e. } k \in \hat{\mathbb{R}}^{d} \backslash N_{F}^{(j)} .
$$

It is easy to see that $T_{F, G}^{(j)}$ is symmetric [note that $D\left(T_{F, G}^{(j)}\right)$ is dense in $L^{2}\left(\mathbb{R}^{d}\right)$, since $C_{0}^{\infty}\left(\hat{\mathbb{R}}^{d} \backslash N_{F}^{(j)}\right)$ is dense in $\left.L^{2}\left(\hat{\mathbb{R}}^{d}\right)\right]$.

Lemma 11. 1 For all $t \in \mathbb{R}$ and $\psi \in D\left(T_{F, G}^{(j)}\right)$, one has $e^{-i t H_{F}} \psi \in D\left(T_{F, G}^{(j)}\right)$.

Proof: We have by the functional calculus $\mathcal{F} e^{-i t H_{F}} \mathcal{F}^{-1}=e^{-i t M_{F}}$, where $M_{F}$ is the multiplication operator by the function $F$. It is easy to see that, for all $u \in C_{0}^{1}\left(\hat{\mathbb{R}}^{d} \backslash N_{F}^{(j)}\right)$, $e^{-i t M_{F}} u \in C_{0}^{1}\left(\hat{\mathbb{R}}^{d} \backslash N_{F}^{(j)}\right)$. Thus the assertion follows.

For the functions $F$ and $G$ as above, the operator

$$
C_{F, G}^{(j)}:=\left[G(\mathbf{p})+G(\mathbf{p})^{*}\right] F_{j}^{(1)}
$$

is bounded and self-adjoint. 
Proposition 11.2 Let $F$ and $G$ be as above. Then $\left(T_{F, G}^{(j)}, H_{F}, K_{C_{F, G}^{(j)}}\right)$ obeys the $G W W R$.

Proof: By using the Fourier transform, it is easy to see that

$$
q_{j} e^{-i t H_{F}} \psi=e^{-i t H_{F}} q_{j} \psi+t e^{-i t H_{F}} F_{j}^{(1)} \psi
$$

for all $\psi \in D\left(q_{j}\right) \cap D\left(F_{j}^{(1)}\right)$, which, together with Lemma 11. 1, implies that

$$
\begin{aligned}
& G(\mathbf{p}) q_{j} e^{-i t H_{F}} \psi=e^{-i t H_{F}} G(\mathbf{p}) q_{j} \psi+t e^{-i t H_{F}} G(\mathbf{p}) F_{j}^{(1)} \psi, \\
& q_{j} G(\mathbf{p})^{*} e^{-i t H_{F}} \psi=e^{-i t H_{F}} q_{j} G(\mathbf{p})^{*} \psi+t e^{-i t H_{F}} F_{j}^{(1)} G(\mathbf{p})^{*} \psi
\end{aligned}
$$

for all $\psi \in D\left(T_{F, G}^{(j)}\right)$. Adding these equations, we obtain

$$
T_{F, G}^{(j)} e^{-i t H_{F}} \psi=e^{-i t H_{F}} T_{F, G}^{(j)} \psi+t e^{-i t H_{F}} C_{F, G}^{(j)} \psi .
$$

Thus the desired result follows.

Example 11. 1 (The free Hamiltonian of a nonrelativistic quantum particle) Consider the case where $F(k)=k^{2} /(2 m)(m>0$ is a constant denoting the mass of a quantum particle) and $G(k)=v(k) / \partial_{j} F(k)=m v(k) k_{j}^{-1}$ with $v \in C^{1}\left(\hat{\mathbb{R}}^{d}\right)$ being bounded. Then

$$
\begin{aligned}
& H_{F}=H_{\mathrm{NR}}:=-\frac{\Delta}{2 m}, \quad T_{F, G}^{(j)}=T_{v}^{(j)}:=m\left(v(\mathbf{p}) p_{j}^{-1} q_{j}+q_{j} v(\mathbf{p})^{*} p_{j}^{-1}\right), \\
& C_{F, G}^{(j)}=C_{v}:=v(\mathbf{p})+v(\mathbf{p})^{*}
\end{aligned}
$$

with $N_{F}^{(j)}=\left\{k \in \hat{\mathbb{R}}^{d} \mid k_{j}=0\right\}$ (hence its $d$-dimensional Lebesgue measure is zero), where

$$
\Delta:=\sum_{j=1}^{d} D_{j}^{2}
$$

is the $d$-dimensional generalized Laplacian on $L^{2}\left(\mathbb{R}^{d}\right)$. Then $\left(T_{v}^{(j)}, H_{\mathrm{NR}}, K_{C_{v}}\right)$ obeys the GWWR. The operator $T_{v}^{(j)}$ with $d=1, m=1 / 2$ and $v=1 / 2$ is just the Aharonov- Bohm time operator mentioned in Example 10.1).

Example 11. 2 (The free Hamiltonian of a relativistic quantum particle) Consider the case where $F(k)=\sqrt{k^{2}+m^{2}}$ with $m>0$ (a constant). Let $G(k)=v(k) / \partial_{j} F(k)=$ $v(k) \sqrt{k^{2}+m^{2}} k_{j}^{-1}$. Then

$$
\begin{aligned}
& H_{F}=H_{\mathrm{rel}}:=\sqrt{-\Delta+m^{2}}, \\
& T_{F, G}^{(j)}=T_{\mathrm{rel}}^{(j)}:=v(\mathbf{p}) \sqrt{-\Delta+m^{2}} p_{j}^{-1} q_{j}+q_{j} v(\mathbf{p})^{*} \sqrt{-\Delta+m^{2}} p_{j}^{-1}, \\
& C_{F, G}^{(j)}=C_{v}
\end{aligned}
$$

with $N_{F}^{(j)}=\left\{k \in \hat{\mathbb{R}}^{d} \mid k_{j}=0\right\}$. Then $\left(T_{\text {rel }}^{(j)}, H_{\text {rel }}, K_{C_{v}}\right)$ obeys the GWWR. 
Example 11. 3 (Powers of the $d$-dimensional Laplacian) Let $\alpha>0$ be a constant and consider the case where

$$
F(k)=F_{\alpha}(k):=|k|^{2 \alpha}, \quad k \in \hat{\mathbb{R}}^{d} .
$$

In this case we have

$$
H_{F_{\alpha}}=(-\Delta)^{\alpha}
$$

Let $M_{j}:=\mathbb{R}^{d} \backslash\left\{k \in \hat{\mathbb{R}}^{d} \mid k_{j}=0\right\}$ and

$$
T_{j}^{(\alpha)}:=\frac{1}{2 \alpha}\left\{v(\mathbf{p})(-\Delta)^{-\alpha+1} p_{j}^{-1} q_{j}+q_{j} v(\mathbf{p})^{*}(-\Delta)^{-\alpha+1} p_{j}^{-1}\right\},
$$

with $D\left(T_{j}^{(\alpha)}\right):=\mathcal{F}^{-1} C_{0}^{1}\left(M_{j}\right)$. Then $\left(T_{j}^{(\alpha)}, H_{F_{\alpha}}, K_{C_{v}}\right)$ obeys the GWWR.

\subsection{Abstract Dirac operators}

Let $\mathcal{K}$ be a Hilbert space and $A_{j}(j=1, \cdots, d)$ and $B$ be bounded self-adjoint operators satisfying the anticommutation relations

$$
\begin{aligned}
& \left\{A_{j}, A_{l}\right\}=2 \delta_{j l}, \quad\left\{A_{j}, B\right\}=0, \\
& B^{2}=I, \quad j, l=1, \cdots, d,
\end{aligned}
$$

where $\{X, Y\}:=X Y+Y X$. Let $M$ be a strictly positive, continuously differentiable function on $\mathbb{R}^{d}$ such that $\partial_{j} M$ is bounded $(j=1, \cdots, d)$ and the set

$$
N_{j}:=\left\{k \in \hat{\mathbb{R}}^{d} \mid k_{j}+M(k)\left(\partial_{j} M\right)(k)=0\right\}
$$

is closed with Lebesuge measure zero. Then the operator

$$
p_{j}(M):=p_{j}+M(\mathbf{p})\left(\partial_{j} M\right)(\mathbf{p})
$$

is injective and $\mathcal{F}^{-1} C_{0}^{\infty}\left(\hat{\mathbb{R}}^{d} \backslash N_{j}\right) \subset D\left(p_{j}(M)^{-1}\right)$.

We define an operator of Dirac type

$$
H_{\mathrm{D}}:=\sum_{j=1}^{d} A_{j} \otimes p_{j}+B \otimes M(\mathbf{p})
$$

acting on $\mathcal{K} \otimes L^{2}\left(\mathbb{R}^{d}\right)$. This is an abstract Dirac operator.

Example 11. 4 The usual free Dirac operator [22, p.7] is given by taking $d=3, \mathcal{K}=$ $\mathbb{C}^{4}, A_{j}=\alpha_{j}, M=m$ (a positive constant), $B=\beta$ with $\alpha_{j}$ and $\beta$ being $4 \times 4$-Hermitian matrices satisfying $\left\{\alpha_{j}, \alpha_{l}\right\}=2 \delta_{j l},\left\{\beta, \alpha_{j}\right\}=0, \beta^{2}=I, j, l=1,2,3$.

By a general theorem [3, Theorem 4.3], $H_{\mathrm{D}}$ is self-adjoint and

$$
H_{\mathrm{D}}^{2}=I \otimes\left(-\Delta+M(\mathbf{p})^{2}\right) .
$$


In what follows, under the natural identification $\mathcal{K} \otimes L^{2}\left(\mathbb{R}^{d}\right)=L^{2}\left(\mathbb{R}^{d} ; \mathcal{K}\right)$ (the Hilbert space of $\mathcal{K}$-valued square integrable functions on $\left.\mathbb{R}^{d}\right)$, we write $A_{j} \otimes p_{j}$ as $A_{j} p_{j}$. Let $v \in C^{1}\left(\hat{\mathbb{R}}^{d}\right)$ be bounded and

$$
T_{j}:=v(\mathbf{p}) H_{\mathrm{D}} p_{j}(M)^{-1} q_{j}+q_{j} v(\mathbf{p})^{*} H_{\mathrm{D}} p_{j}(M)^{-1}
$$

with $D\left(T_{j}\right):=\mathcal{K} \hat{\otimes} \mathcal{F}^{-1} C_{0}^{\infty}\left(\hat{\mathbb{R}}^{d} \backslash N_{j}\right)$, where $\hat{\otimes}$ denotes algebraic tensor product.

Theorem 11. 3 The triple $\left(T_{j}, H_{\mathrm{D}}, K_{C_{v}}\right)$ obeys the $G W W R$.

Proof : The self-adjoint operator $L:=\left(-\Delta+M(\mathbf{p})^{2}\right)^{1 / 2}$ is strictly positive. Hence it has a bounded inverse. Let

$$
U:=\frac{1}{\sqrt{2}} B\left(H_{\mathrm{D}}+B L\right) L^{-1 / 2}\left(L+M(\mathbf{p})^{-1 / 2} .\right.
$$

Then $U$ is unitary and

$$
U H_{\mathrm{D}} U^{*}=B L .
$$

(This is an abstract structure of the usual free Dirac operator, see, $[22, \S 1.4]$.) We also have

$$
\hat{T}_{j}:=U T_{j} U^{*}=v(\mathbf{p}) B L p_{j}(M)^{-1} q_{j}+q_{j} v(\mathbf{p})^{*} B L p_{j}(M)^{-1}
$$

with $D\left(\hat{T}_{j}\right):=U D\left(T_{j}\right)$ (note that $U C_{v}=C_{v} U$ ). Using the Fourier analysis, we can show that $\left(\hat{T}_{j}, B L, K_{C_{v}}\right)$ obeys the GWWR. This implies that $\left(T_{j}, H_{\mathrm{D}}, K_{C_{v}}\right)$ obeys the GWWR.

Remark 11.1 We can apply general results established in Sections 2-10 to the Hamiltonians $H_{F}, H_{\mathrm{NR}}, H_{\mathrm{rel}}, H_{F_{\alpha}}$ and the Dirac operator $H_{\mathrm{D}}$. In particular, we can derive decay estimates (in time $t$ ) for transition probability amplitudes of states with these Hamiltonians. But we do not write down them here.

\section{Representations of the GWWR in Fock Spaces}

In this section we show that, given a triple obeying the GWWR in a Hilbert space $\mathcal{H}$, there exist triples obeying the GWWR in Fock spaces (full Fock spaces, boson Fock spaces, fermion Fock spaces) over $\mathcal{H}$.

\subsection{Tensor Representations of the GWWR}

Let $n \in \mathbb{N}$ and $\mathcal{H}_{j}(j=1, \cdots, n, n \in \mathbb{N})$ be a Hilbert space. Suppose that $\left(T_{j}, H_{j}, K_{C_{j}}\right)$ obeys the GWWR in $\mathcal{H}_{j}$ such that $T_{j}$ is closed symmetric. We consider an operator

$$
L:=\overline{\sum_{j=1}^{n} \underbrace{I \otimes \cdots \otimes I \otimes H_{j} \otimes I \otimes \cdots \otimes I}_{n} \mid \hat{\otimes}_{j=1}^{n} D\left(H_{j}\right)},
$$


where $\hat{\otimes}$ denotes algebraic tensor product. It is well known that $L$ is self-adjoint $[19$, $\S$ VIII.10] and

$$
e^{-i t L}=e^{-i t H_{1}} \otimes \cdots \otimes e^{-i t H_{n}}, \quad t \in \mathbb{R} .
$$

Let

$$
\begin{aligned}
\widetilde{T}_{j} & :=\underbrace{I \otimes \cdots \otimes I \otimes T_{j} \otimes I \otimes \cdots \otimes I}_{n}, \\
\widetilde{C}_{j} & :=\underbrace{I \otimes \cdots \otimes I \otimes C_{j} \otimes I \otimes \cdots \otimes I}_{n} .
\end{aligned}
$$

Proposition 12. 1 For all $j=1, \cdots, n,\left(\widetilde{T}_{j}, L, K_{\widetilde{C}_{j}}\right)$ obeys the $G W W R$.

Proof : Let $\Psi=\psi_{1} \otimes \cdots \otimes \psi_{n}$ with $\psi_{j} \in D\left(T_{j}\right), j=1, \cdots, n$. Then, for all $t \in \mathbb{R}$, $e^{-i t L} \Psi=\otimes_{l=1}^{n} e^{-i t H_{l}} \psi_{l} \in \hat{\otimes}_{l=1}^{n} D\left(T_{l}\right)$ and

$$
\begin{aligned}
\widetilde{T}_{j} e^{-i t L} \Psi & =e^{-i t H_{1}} \psi_{1} \otimes \cdots e^{-i t H_{j-1}} \psi_{j-1} \otimes T_{j} e^{-i t H_{j}} \psi_{j} \otimes e^{-i t H_{j+1}} \psi_{j+1} \cdots \otimes \otimes e^{-i t H_{n}} \psi_{n} \\
& =e^{-i t H_{1}} \psi_{1} \otimes \cdots \otimes e^{-i t H_{j}}\left(T_{j}+t C_{j}\right) \psi_{j} \otimes \cdots \otimes e^{-i t H_{n}} \psi_{n} \\
& =e^{-i t L}\left(\widetilde{T}_{j}+t \widetilde{C}_{j}\right) \Psi .
\end{aligned}
$$

Since $\hat{\otimes}_{j=1}^{n} D\left(T_{j}\right)$ is a core of $\widetilde{T}_{j}$, the last equality extends to all $\Psi \in D\left(\widetilde{T}_{j}\right)$ with $e^{-i t L} \Psi \in$ $D\left(\widetilde{T}_{j}\right)$. Hence $\left(\widetilde{T}_{j}, L, K_{\widetilde{C}_{j}}\right)$ obeys the GWWR.

\subsection{Constructions of triples obeying the GWWR in Fock S- paces}

Let $\mathcal{H}$ be a Hilbert space. Then the full Fock space over $\mathcal{H}$ is defined by

$$
\mathcal{F}(\mathcal{H}):=\oplus_{n=0}^{\infty} \otimes^{n} \mathcal{H}
$$

with $\otimes^{0} \mathcal{H}:=\mathbb{C}$ (see, e.g., [7, §5.2] or [19, §II.4, §VIII.10] for Fock space theory). For a densely defined closed linear operator $A$ on $\mathcal{H}$ and $n \in\{0\} \cup \mathbb{N}$, we define a linear operator $A_{\Sigma}^{(n)}$ on $\otimes^{n} \mathcal{H}$ as follows:

$$
\begin{aligned}
A_{\Sigma}^{(0)} & :=0 \\
A_{\Sigma}^{(n)} & :=\underbrace{(\sum_{j=1}^{n} \underbrace{I \otimes \cdots \otimes I \otimes \underbrace{A}_{\text {-th }} \otimes I \otimes \cdots \otimes I}) \mid \hat{\otimes}^{n} D(A)}_{n}
\end{aligned}
$$

for $n \geq 1$. Then the operator

$$
d \Gamma(A):=\oplus_{n=0}^{\infty} A_{\Sigma}^{(n)},
$$

called the second quantization of $A$, is densely defined and closed. If $A$ is self-adjoint, then so is $d \Gamma(A)$.

Let $H$ be a self-adjoint operator on $\mathcal{H}, T$ be a closed symmetric operator on $\mathcal{H}$ and $C \in \mathrm{B}(\mathcal{H})$ be self-adjoint. In what follows we take the following hypothesis: 
Hypothesis (I) The triple $\left(T, H, K_{C}\right)$ obeys the GWWR in $\mathcal{H}$.

We define

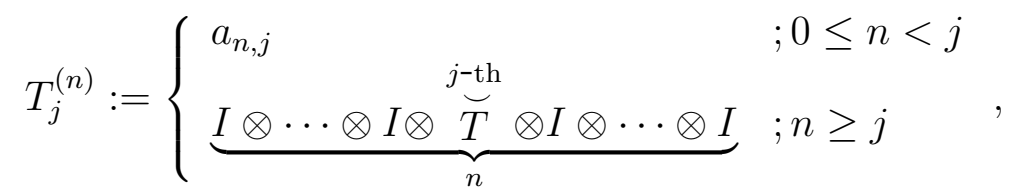

where $a_{n, j}$ is an arbitrary real constant. For $j \in \mathbb{N}$, we define a linear operator $T_{j}$ on $\mathcal{F}(\mathcal{H})$ by

$$
T_{j}:=\oplus_{n=0}^{\infty} T_{j}^{(n)} .
$$

Then $T_{j}$ is a closed symmetric operator. Let

$$
C_{j}^{(n)}:= \begin{cases}0 & ; 0 \leq n<j \\ \underbrace{I \otimes \cdots \otimes I \otimes \stackrel{j-t h}{C}^{I \otimes I \otimes \cdots \otimes I}}_{n} & ; n \geq j\end{cases}
$$

and

$$
C_{j}:=\oplus_{n=0}^{\infty} C_{j}^{(n)} .
$$

Then $C_{j}$ is a bounded self-adjoint operator on $\mathcal{F}(\mathcal{H})$.

Proposition 12.2 Under Hypothesis (I), for all $j \in \mathbb{N},\left(T_{j}, d \Gamma(H), K_{C_{j}}\right)$ obeys the GWWR.

Proof : It follows from Proposition 12.1 that, for all $n,\left(T_{j}^{(n)}, H_{\Sigma}^{(n)}, K_{C_{j}^{(n)}}\right)$ obeys the GWWR. It is easy to see that this implies the desired result.

We next construct a triple obeying the GWWR in $\mathcal{F}(\mathcal{H})$ which is reduced by the boson Fock space over $\mathcal{H}$

$$
\mathcal{F}_{\mathrm{b}}(\mathcal{H}):=\oplus_{n=0}^{\infty} \otimes_{\mathrm{s}}^{n} \mathcal{H},
$$

where $\otimes_{\mathrm{s}}^{n} \mathcal{H}$ denotes the $n$-fold symmetric tensor product of $\mathcal{H}$, and the fermion Fock space over $\mathcal{H}$

$$
\mathcal{F}_{\mathrm{f}}(\mathcal{H}):=\oplus_{n=0}^{\infty} \otimes_{\text {as }}^{n} \mathcal{H},
$$

where $\otimes_{\text {as }}^{n} \mathcal{H}$ denotes the $n$-fold antisymmetric tensor product of $\mathcal{H}$. The operator

$$
\hat{N}:=d \Gamma(I)
$$

on $\mathcal{F}(\mathcal{H})$ is called the number operator. The vector

$$
\Omega:=\{1,0,0, \cdots\} \in \mathcal{F}(\mathcal{H})
$$

is called the Fock vacuum. We denote by $P_{0}$ the orthogonal projection onto the onedimensional subspace $\{z \Omega \mid z \in \mathbb{C}\}$. We set

$$
Q_{0}:=I-P_{0} .
$$

Let

$$
S:=Q_{0} \hat{N}^{-1 / 2} d \Gamma(C) \hat{N}^{-1 / 2} Q_{0}
$$


Lemma 12.3 The operator $S$ is bounded and self-adjoint with $\|S\| \leq\|C\|$.

Proof: It is easy to see that, for all $\Psi \in D(S),(S \Psi)^{(0)}=0$ and, for $n \geq 1$,

$$
(S \Psi)^{(n)}=\frac{(d \Gamma(C) \Psi)^{(n)}}{n} .
$$

Hence $\left\|(S \Psi)^{(n)}\right\| \leq\|C\|\left\|\Psi^{(n)}\right\|$, which implies that $\|S \Psi\|^{2} \leq\|C\|^{2}\|\Psi\|^{2}$. Hence $S$ is bounded with $\|S\| \leq\|C\|$ and $D(S)=\mathcal{F}(\mathcal{H})$. It is easy to see that $S$ is symmetric.

Let

$$
\mathcal{D}_{T}:=\oplus_{n=0}^{\infty} \hat{\otimes}^{n} D(T)
$$

and

$$
\tau(T):=\left(Q_{0} \hat{N}^{-1 / 2} d \Gamma(T) \hat{N}^{-1 / 2} Q_{0}\right) \mid \mathcal{D}_{T} .
$$

Then $\tau(T)$ is symmetric.

Theorem 12. 4 Assume Hypothesis (I). Then $\left(\tau(T), d \Gamma(H), K_{S}\right)$ obeys the $G W W R$.

Proof : For all $\Psi \in \mathcal{D}_{T}, \hat{N}^{-1 / 2} Q_{0} \Psi \in \mathcal{D}_{T}$, since

$$
\left(\hat{N}^{-1 / 2} Q_{0} \Psi\right)^{(0)}=0, \quad\left(\hat{N}^{-1 / 2} Q_{0} \Psi\right)^{(n)}=n^{-1 / 2} \Psi^{(n)}, \quad n \geq 1 .
$$

Using this property and the easily proven fact that $e^{-i t d \Gamma(H)} \hat{N}^{-1 / 2} Q_{0}=\hat{N}^{-1 / 2} Q_{0} e^{-i t d \Gamma(H)}$, we have by direct computations

$$
d \Gamma(T) \hat{N}^{-1 / 2} Q_{0} e^{-i t d \Gamma(H)} \Psi=e^{-i t d \Gamma(H)} d \Gamma(T) \hat{N}^{-1 / 2} Q_{0} \Psi+t e^{-i t d \Gamma(H)} d \Gamma(C) \hat{N}^{-1 / 2} Q_{0} \Psi .
$$

Operating $Q_{0} \hat{N}^{-1 / 2}$ to the both sides, we obtain

$$
\tau(T) e^{-i t d \Gamma(H)} \Psi=e^{-i t d \Gamma(H)} \tau(T) \Psi+t e^{-i t d \Gamma(H)} S \Psi,
$$

which implies the desired result.

Remark 12.1 Applying the results in Section 8, we can derive decay estimates for $\left|\left\langle\Phi, e^{-i t d \Gamma(H)} \Psi\right\rangle\right|(t \in \mathbb{R})$ for vectors $\Phi, \Psi$ in suitable subspaces. But we do not write down them here. The same applies to other results implied by the abstract theory.

It is easy to see that $d \Gamma(H), \tau(T)$ and $S$ are reduced by $\mathcal{F}_{\#}(\mathcal{H})(\#=\mathrm{b}, \mathrm{f})$. Hence Theorem 12. 4 holds for the reduced parts of them too.

It is more interesting and important to construct generalized time operators of perturbed Hamiltoinians of the form $d \Gamma(H)+V$ on the boson Fock space $\mathcal{F}_{\mathrm{b}}(\mathcal{H})$ or the fermion Fock space $\mathcal{F}_{\mathrm{f}}(\mathcal{H})$ with $V$ a symmetric operator. For this purpose, the method given in Section 2.4 can be applied. But in the present paper we leave this problem for future study. 


\section{References}

[1] Y. Aharonov and D. Bohm, Significance of electromagnetic potentials in the quantum theory, Phys. Rev. 115 (1959), 485-491.

[2] Y. Aharonov and D. Bohm, Time in the quantum theory and the uncertainty relation for time and energy, Phys. Rev. 122 (1961), 1649-1658.

[3] A. Arai, Characterization of anticommutativity of self-adjoint operators in connection with Clifford algebra and applications, Integr. Equat. Oper. Th. 17 (1993), 451-463.

[4] A. Arai, Hilbert Spaces and Quantum Mechanics, Kyoritsu-shuppan, Tokyo, 1997 (in Japanese).

[5] A. Arai, Representation-theoretic aspects of two-dimensional quantum systems in singular vector potentials: canonical commutation relations, quantum algebras, and reduction to lattice quantum systems, J. Math. Phys. 39 (1998), 2476-2498.

[6] A. Arai, Mathematical Principles of Quantum Phenomena, Asakura-shoten, Tokyo, 2005, to be published (in Japanese).

[7] O. Bratteli and D. W. Robinson, Operator Algebras and Quantum Statistical Mechanics 2, Second Edition, Springer, Berlin, Heidelberg, 1997.

[8] B. Fuglede, On the relation $P Q-Q P=-i \mathrm{I}_{\mathrm{d}}$, Math. Scand. 20 (1967), 79-88.

[9] S. T. Kuroda, Spectral Theory II, Iwanami-shoten, 1979 (in Japanese).

[10] L. Mandel and E. Wolf, Optical Coherence and Quantum Optics, Cambridge University Press, Cambridge, 1995.

[11] M. Miyamoto, A generalized Weyl relation approach to the time operator and its connection to the survival probability, J. Math. Phys. 42 (2001), 1038-1052.

[12] M. Miyamoto, Characteristic dedcay of the autocorrelation functions prescribed by the Aharonov-Bohm time operator, arXiv:quant-ph/0105033v2, 2001.

[13] M. Miyamoto, The various power decays of the survival probability at long times for free quantum particle, J. Phys. A 35 (2002), 7159-7171.

[14] M. Miyamoto, Difference between the decay forms of the survival and nonescape probabilities, arXiv:quant-ph/0207067v2, 2002.

[15] J. G. Muga, R. Sala Mayato and I. L. Egusquiza (Eds.), Time in Quantum Mechanics, Springer, 2002.

[16] J. von Neumann, Die Eindeutigkeit der Schrödingerschen Operatoren, Math. Ann. 104 (1931), 570-578.

[17] P. Pfeifer and J. Fröhlich, Generalized time-energy uncertainty relations and bounds on lifetimes of resonances, Rev. Mod. Phys. 67 (1995), 759-779. 
[18] C. R. Putnam, Commutation Properties of Hilbert Space Operators, Springer, Berlin, 1967.

[19] M. Reed and B. Simon, Methods of Modern Mathematical Physics I: Functional Analysis, Academic Press, New York, 1972.

[20] M. Reed and B. Simon, Methods of Modern Mathematical Physics III: Scattering Theory, Academic Press, New York, 1979.

[21] K. Schmüdgen, On the Heisenberg commutation relation. I, J. Funct. Anal. 50 (1983), 8-49.

[22] B. Thaller, The Dirac Equation, Springer, Berlin Heidelberg, 1992.

[23] E. P. Wigner, On the time-energy uncertainty relation, in Aspects of Quantum Theory (edited by A. Salam and E. P. Wigner, Cambridge University, Cambridge, UK), 237247. 